\title{
PRESENTATIONS OF INVERSE SEMIGROUPS, THEIR KERNELS AND EXTENSIONS
}

\author{
CATARINA CARVALHO, ROBERT D. GRAY ${ }^{\otimes}$ and NIK RUSKUC
}

(Received 20 May 2010; accepted 28 October 2010)

Communicated by M. G. Jackson

\begin{abstract}
Let $S$ be an inverse semigroup and let $\pi: S \rightarrow T$ be a surjective homomorphism with kernel $K$. We show how to obtain a presentation for $K$ from a presentation for $S$, and vice versa. We then investigate the relationship between the properties of $S, K$ and $T$, focusing mainly on finiteness conditions. In particular we consider finite presentability, solubility of the word problem, residual finiteness, and the homological finiteness property $\mathrm{FP}_{n}$. Our results extend several classical results from combinatorial group theory concerning group extensions to inverse semigroups. Examples are also provided that highlight the differences with the special case of groups.
\end{abstract}

2010 Mathematics subject classification: primary 20M18; secondary 20M05.

Keywords and phrases: inverse semigroup presentations, Reidemeister-Schreier, kernel, finiteness conditions.

\section{Introduction}

Let $K \rightarrow G \rightarrow T$ be a short exact sequence of groups, that is, $G$ is an extension of $K$ by $T$. There are several well-known results in combinatorial group theory relating the properties of $G, K$ and $T$. For example, we have the following results (see, for instance, [20, Ch. V]).

(i) If $K$ is finite, then $G$ is finitely presented if and only if $T$ is finitely presented.

(ii) If $T$ is finite, then $G$ is finitely presented if and only if $K$ is finitely presented.

Each of these results is a consequence of the fact that finite presentability is a quasiisometry invariant of finitely generated groups: in (i), finiteness of $K$ implies that $G$ and $T$ are quasiisometric, while, in (ii), finiteness of $T$ implies that $G$ and $K$ are quasiisometric. (Of course, (ii) is also a corollary of the classical ReidemeisterSchreier theorem for subgroups of finite index.) Consequently, for finitely generated groups the analogous results to those above hold with finite presentability replaced

Carvalho was supported by FCT grant SFRH/BPD/26216/2006. Part of this work was done while Gray was an EPSRC Postdoctoral Research Fellow at the University of St Andrews, Scotland. Gray was also partially supported by FCT and FEDER, project POCTI-ISFL-1-143 of the Centro de Álgebra da Universidade de Lisboa, and by the project PTDC/MAT/69514/2006.

(C) 2011 Australian Mathematical Publishing Association Inc. 1446-7887/2011 \$16.00 
by any other quasiisometry invariant of groups including, among others, the property $\mathrm{F}_{n}$ (which generalises finite generation and presentability to arbitrary dimensions), the related homological finiteness property $\mathrm{FP}_{n}$, and the property of being finitely presented with soluble word problem; see [20, p. 115] and [1, 2].

In the more general context of semigroup theory, homomorphisms are studied via congruences. In the case of groups, the study of congruences reduces to the consideration of normal subgroups, but for semigroups in general no corresponding reduction to substructures is possible. So in general it does not make sense to consider analogues of the results for groups mentioned above for arbitrary semigroups. However, such questions do make sense for inverse semigroups via the classical kernel-trace description of congruences. See [28, Ch. 5] for background on this theory.

Following, for example, [19], a pair $(S, \pi)$, where $S$ is an inverse semigroup containing $K$ as a subsemigroup and $\pi$ is a homomorphism of $S$ onto an inverse semigroup $T$ such that $\pi^{-1}(E(T))=K$, where $E(T)$ is the set of idempotents of $T$, is called an extension of $K$ by $T$. Of course, when $S$ is a group this puts us exactly in the situation discussed above. Extensions of inverse semigroups are an important and well-studied area in the subject, with recent examples including [9, 23]. The vast majority of research in this area has been concerned with proving structural results for certain special kinds of extension. The results presented in this paper do not fall into that category.

In this paper, our primary interest is in combinatorial inverse semigroup theory, that is, the study of inverse semigroups using presentations. Combinatorial inverse semigroup theory is a subject in its own right, with a substantial body of literature; see, for instance, [10, 27, 34, 41, 42, 44]. Other papers where finiteness conditions of inverse semigroups are considered include [21,37]. Our interest here is in studying extensions of inverse semigroups, but within the context of combinatorial inverse semigroup theory. As a necessary first step, we shall give methods for writing down presentations for extensions of inverse semigroups, and conversely given a presentation for an extension to determine a presentation for the kernel $K$. We then apply these results to investigate the following very general question.

Let $S$ be an inverse semigroup and suppose that $(S, \pi)$ is an extension of $K$ by $T$. How are the properties of $S, K$ and $T$ related to one another?

The results that we present here serve as an initial investigation of this question, and we begin by considering to what extent the results mentioned above concerning finiteness properties of groups hold true for inverse semigroup extensions. Unsurprisingly, in general we shall see that the results do not carry across to inverse semigroups without modification, and so we shall end up with a mixture of counterexamples for results in general, theorems in certain cases (often with some additional restriction placed on the nature of the homomorphism $\pi$ ), and we also present several open problems, which highlight the limits of our techniques at present, and suggest possible future directions for research.

The paper is laid out as follows. Our main results concern presentations, and are given in Sections 3 and 4. We go on to apply these results in Section 5 to investigate 
the relationship between finiteness properties of inverse semigroups and their kernels, specifically we obtain results for finite generation and presentability, solubility of the word problem, and residual finiteness. Finally, in Section 6, we consider the relationship between properties of inverse semigroups and their homomorphic images, under the assumption that the kernel is finite, and in particular make some observations about finite presentability and the homological finiteness property $\mathrm{FP}_{n}$.

The methods used throughout are combinatorial. In particular we make use of the Reidemeister-Schreier type rewriting methods for semigroups originally introduced in [16]. As mentioned above, in the special case of groups, many of these results may be explained geometrically (via the notion of quasiisometry). It would be interesting to know whether there are corresponding geometric interpretations more generally for the results we present here for inverse semigroups. It is possible that the topological approach to inverse semigroup presentations developed in [41] might shed some light on this.

\section{Preliminaries}

2.1. Homomorphisms, congruences, kernels and extensions. We begin by introducing the basic concepts used in this paper. For a more detailed introduction to general notions in inverse semigroup theory, we refer the reader to [26, 28]. Let $S$ be an inverse semigroup and let $E(S)$ denote the set of idempotents of $S$. The elements of $S$ carry a natural partial order $\leq$ where, given any two elements $s, t \in S$, we say that $s \leq t$ if there exists an idempotent $e \in E(S)$ such that $s=t e$. This order is compatible both with multiplication and inversion. Furthermore, when restricted to the set of idempotents this order becomes $e \leq f$ if and only if $e f=e$ for all $e, f \in E(S)$. Since $E(S)$ is a commutative subsemigroup of $S,(E(S), \leq)$ forms a meet semilattice (where the meet operation is multiplication).

We now fix some notation that will remain in force throughout this paper. Let $\pi: S \rightarrow T$ be a surjective homomorphism of inverse semigroups. The kernel of this homomorphism is

$$
K=\operatorname{ker} \pi=\pi^{-1}(E(T))=\bigcup_{e \in E(T)} C_{e},
$$

where $E(T)$ is the set of idempotents of $T$ and

$$
C_{t}=\{s \in S: s \pi=t\}=\pi^{-1}(t) \quad \forall t \in T .
$$

Clearly $K$ is an inverse subsemigroup of $S$ and $E(K)=E(S)$, that is, it is full. For every $t \in T$, let

$$
E_{t}=\left\{s^{-1} s: s \in C_{t}\right\} \subseteq E(S) .
$$

In other words $E_{t}$ denotes the set of all idempotents appearing in $\mathcal{L}$-classes of elements of $\pi^{-1}(t)$, where $\mathcal{L}$ denotes Green's $\mathcal{L}$-relation (see [26, Ch. 2] for more on Green's relations). Next, for every $t \in T$, we shall fix a subset $F_{t} \subseteq E_{t}$ such that

$$
\left(\forall e \in E_{t}\right)\left(\exists f_{e} \in F_{t}\right) \quad e \leq f_{e} .
$$


In other words, $F_{t}$ is a set of idempotents that 'covers' all the idempotents $E_{t}$ in the sense that every element of $E_{t}$ lies below some element from $F_{t}$. Of course, such a set $F_{t}$ always exists, since if necessary we can take $F_{t}=E_{t}$.

As we shall see later on in Section 5, the question of whether or not the sets $F_{t}$ may be chosen to be finite will influence whether or not finite generation or presentability is preserved when passing between $S$ and $K$. Specifically, we shall see in Proposition 5.2 that there is a surjective homomorphism from the free inverse monoid of rank two onto the finite cyclic group of order three whose kernel is not finitely generated. Thus, although it may not be completely transparent at present, the introduction of the set $F_{t}$ here is necessary in order that positive results about preservation of finiteness properties may be obtained in Section 5 .

For each $t \in T$ and $e \in F_{t}$, fix a representative $s_{e, t} \in C_{t}$ such that $e=s_{e, t}^{-1} s_{e, t}$. Then define

$$
Q_{t}=\left\{s_{e, t}: e \in F_{t}\right\} \subseteq C_{t} \quad \text { and } \quad Q=\bigcup_{t \in T} Q_{t} .
$$

Note that $\left|Q_{t}\right|=\left|F_{t}\right|$ for each $t \in T$, so if $F_{t}$ is finite for all $t \in T$, and $T$ is finite, then $Q$ is finite.

The following fundamental lemma will be used throughout the paper.

Lemma 2.1. With the notation above, every element $s \in S$ can be decomposed in the form $s=u q$ for some $u \in K$ and $q \in Q_{t}$ with $\pi(s)=t$ and $u=s q^{-1}$.

Proof. From $s \in C_{t}$ and (2.1), it follows that $s^{-1} s \in E_{t}$. By definition of $F_{t}$ there exists $e \in F_{t}$ such that $s^{-1} s \leq e$. Therefore, for $q=s_{e, t} \in Q$ we have $\pi(q)=t=\pi(s)$ and $s^{-1} s \leq q^{-1} q$. Now, $s^{-1} s q^{-1} q=s^{-1} s$, which implies that $s s^{-1} s q^{-1} q=s s^{-1} s$, and so $s q^{-1} q=s$. Also, from $\pi(q)=\pi(s)$, it follows that $\pi\left(q q^{-1}\right)=\pi\left(s q^{-1}\right)$, and so $s q^{-1} \in K$ since $\pi\left(q q^{-1}\right)$ is an idempotent.

Corollary 2.2. Let $S$ be an extension of $K$ by $T$. If $A \subseteq K$ is a generating set for $K$, then, with the notation above, $A \cup Q$ is a generating set for $S$.

It is with respect to the generating set $A \cup Q$ that we give a presentation for $S$ in Section 3 below.

2.2. Inverse semigroup presentations. Let $A$ be a nonempty set, which we call an alphabet. A semigroup presentation is a pair $\langle A \mid R\rangle$, where $R \subseteq A^{+} \times A^{+}$. The elements of $A$ are called generators and the elements of $R$ are called relations. The semigroup defined by the presentation $\langle A \mid R\rangle$ is the semigroup $S=A^{+} / \rho$, where $\rho$ is the smallest congruence on $A^{+}$containing $R$. For $u, v \in A^{+}$we write $u \equiv v$ if $u$ and $v$ are identical words in $A^{+}$and $u=v$ if they represent the same element of $S$. We use $|u|$ to denote the number of letters in the word $u \in A^{+}$, which we call the length of the word $u$. Given $u, v \in A^{+}$we say that $u=v$ is a consequence of $R$ if $u$ can be transformed into $v$ by a finite number of applications of relations from $R$. The semigroup $S$ is said to be finitely generated as a semigroup if $A$ can be chosen to be finite and finitely presented 
if both $A$ and $R$ can be chosen to be finite. Monoid presentations are defined similarly, replacing $A^{+}$by $A^{*}$.

Let $A^{-1}$ be a set disjoint from $A$ and in one-to-one correspondence with $A$ via the map $a \mapsto a^{-1}$. Let $\operatorname{FI}(A)$ denote the free inverse semigroup on a set $A$. Thus $\operatorname{FI}(A)=$ $\left(A \cup A^{-1}\right)^{+} / \rho$ where $\rho$ is the Vagner congruence on the free semigroup $\left(A \cup A^{-1}\right)^{+}$:

$$
\left\{\left(x x^{-1} x, x\right),\left(x x^{-1} y y^{-1}, y y^{-1} x x^{-1}\right): x, y \in\left(A \cup A^{-1}\right)^{+}\right\} .
$$

Here $\left(x^{-1}\right)^{-1}$ should be interpreted as $x$, for $x \in A$, and the map $a \mapsto a^{-1}$ has been extended to words in the natural way. An inverse semigroup presentation is a pair $\langle A \mid R\rangle$, where $R$ is a subset of $\left(A \cup A^{-1}\right)^{+} \times\left(A \cup A^{-1}\right)^{+}$. The inverse semigroup defined by the inverse semigroup presentation $\langle A \mid R\rangle$, is the semigroup $\left(A \cup A^{-1}\right)^{+} / \tau$ where $\tau$ is the congruence on $\left(A \cup A^{-1}\right)^{+}$generated by $\rho \cup R$.

Given an inverse semigroup $S$ there is an important distinction to be made between the question of whether $S$ is finitely presented as an inverse semigroup, and whether $S$ is finitely presented as a semigroup. Indeed, in [40] it was shown that the free inverse semigroup (of any rank) is not finitely presented as a semigroup. Throughout this paper we shall work with inverse semigroups, inverse semigroup presentations, and by finitely presented we shall always mean finitely presented as an inverse semigroup.

\section{A presentation for the extension}

Let $S$ be an inverse semigroup and suppose that $(S, \pi)$ is an extension of $K$ by $T$. Given a presentation $\langle A \mid R\rangle$ for $K$ in this section we show how to obtain a presentation for $S$. We continue using the same notation and conventions introduced in Section 2. We saw in Corollary 2.2 that, viewed as a subset of $S, A \cup Q$ generates $S$. We shall give a presentation for $S$ in terms of this generating set. Since $A \cup Q$ generates $S$, we may extend the mapping $\pi: S \rightarrow T$ to $\pi:\left(\left(A \cup A^{-1}\right) \cup\left(Q \cup Q^{-1}\right)\right)^{+} \rightarrow T$ in the obvious way, and we abuse notation slightly by using the same symbol $\pi$ for both maps.

Before we write down the presentation for $S$ we must first give some definitions. We saw in Lemma 2.1 that every element $s \in S$ can be decomposed (although not necessarily uniquely) in the form $s=u q$ for some $u \in K$ and some $q \in Q_{t}$ where $\pi(s)=t$ and $u=s q^{-1}$. We will say that a word in $\left(\left(A \cup A^{-1}\right) \cup\left(Q \cup Q^{-1}\right)\right)^{*}$ is in normal form, either if it is the empty word, or if it is of the form $w q$ where $w \in\left(A \cup A^{-1}\right)^{*}$ and $q \in Q$ are such that $\pi(w q)=\pi(q)$ and $w q q^{-1}=w$. Let $N$ be the set of words in normal form from $\left(\left(A \cup A^{-1}\right) \cup\left(Q \cup Q^{-1}\right)\right)^{*}$. Note the slightly nonstandard terminology here: normal form would usually suggest a unique set of representatives, which is not the case here, as we now observe.

Lemma 3.1. If $w_{1} q, w_{2} q \in N$, where $w_{1}, w_{2} \in\left(A \cup A^{-1}\right)^{*}$ and $q \in Q$, then the relation $w_{1} q=w_{2} q$ holds in $S$ if and only if $w_{1}=w_{2}$ holds in $K$.

PRoof. As $w_{1} q, w_{2} q$ are in normal form, we know that $w_{1} q q^{-1}=w_{1}$ and $w_{2} q q^{-1}=w_{2}$. It is then clear that $w_{1} q=w_{2} q$ if and only if $w_{1}=w_{2}$. 
It is important to note, however, that it is quite possible that two words $w_{1} q_{1}$ and $w_{2} q_{2}$ can be in normal form, and $w_{1} q_{1}=w_{2} q_{2}$ but $q_{1} \neq q_{2}$.

Our general strategy for obtaining a presentation for $S$ is as follows. First we write down a set of relations that allow us to rewrite an arbitrary word $w$ in the generators $\left(A \cup A^{-1}\right) \cup\left(Q \cup Q^{-1}\right)$ into normal form $\bar{w} q_{w}$. Then given two arbitrary words $w_{1}$ and $w_{2}$ corresponding to the same element of $S$, we rewrite each of them into normal form $\overline{w_{1}} q_{w_{1}}$ and $\overline{w_{2}} q_{w_{2}}$. At this stage we might not have $q_{w_{1}}=q_{w_{2}}$ so in the presentation we also include a set of relations that allow us to rewrite $\overline{w_{1}} q_{w_{1}}$ into ${\overline{w_{1}}}^{\prime} q_{w_{2}}$, which is a word in normal form. Then ${\overline{w_{1}}}^{\prime}=\overline{w_{2}}$ in $K$ by Lemma 3.1, and this can be deduced by applications of relations from $R$.

For each word $w \in\left(\left(A \cup A^{-1}\right) \cup\left(Q \cup Q^{-1}\right)\right)^{+}$, choose and fix a word $\bar{w} \in\left(A \cup A^{-1}\right)^{*}$ and a letter $q_{w} \in Q$ such that $\bar{w} q_{w}$ is in normal form (that is, such that $\bar{w} q_{w} \in N$ ) and $w=\bar{w} q_{w}$ in $S$. Such choices are possible by Lemma 2.1. We extend this notation to the empty word, setting $\overline{1}=1$ and $q_{1}=1$. Next, set $Q^{1}=Q \cup\{1\}$, and define a mapping

$$
f: Q^{1} \times\left(\left(A \cup A^{-1}\right) \cup\left(Q \cup Q^{-1}\right)\right)^{*} \longrightarrow N
$$

by

$$
f(r, w)= \begin{cases}\overline{r w} q_{r w} & \text { if }|w| \leq 1, \\ \overline{r x} f\left(q_{r x}, w^{\prime}\right) & \text { if } w \equiv x w^{\prime} \text { with }\left|w^{\prime}\right| \geq 1,\end{cases}
$$

where $w \equiv x w^{\prime}$ and $x \in\left(A \cup A^{-1}\right) \cup\left(Q \cup Q^{-1}\right)$ while $w^{\prime} \in\left(\left(A \cup A^{-1}\right) \cup\left(Q \cup Q^{-1}\right)\right)^{*}$. It is easy to prove by induction on the length of the word $w$ that $f$ is indeed a mapping from $Q^{1} \times\left(\left(A \cup A^{-1}\right) \cup\left(Q \cup Q^{-1}\right)\right)^{*}$ to $N$. Note that in particular $f(1,1)=1$, which belongs to $N$ by definition. Using this mapping, we are now in a position to state the main result of this section.

THEOREM 3.2. Let $S$ be an inverse semigroup and suppose that $(S, \pi)$ is an extension of $K$ by $T$. Let $\langle A \mid R\rangle$ be a presentation for the kernel $K$. Then, with the notation above, $S$ is defined by a presentation $\langle A, Q \mid R, U\rangle$, where $U$ is the following set of relations

$$
\begin{gathered}
\left\{r a=f(r, a): a \in\left(\left(A \cup A^{-1}\right) \cup\left(Q \cup Q^{-1}\right)\right)^{1}, r \in Q^{1},(r, a) \neq(1,1)\right\} \\
\cup\left\{q r^{-1}=\widehat{q r^{-1}}: q, r \in Q, \pi(q)=\pi(r)\right\},
\end{gathered}
$$

where $\widehat{q r^{-1}}$ is a word in $\left(A \cup A^{-1}\right)^{*}$ representing the element $q r^{-1}$ of $K$ for all $q, r \in Q$.

In particular, if $K$ is finitely presented, and $Q$ is finite, then $S$ is finitely presented.

Clearly the size of $Q$ relates to both the size of the image $T$ (that is, the number of $\pi$-classes), and also the relationship between the homomorphism $\pi$ and the underlying semilattice of idempotents $E(S)$; see Section 5 for further discussion of this. Before proving Theorem 3.2 we need the following lemma.

Lemma 3.3. The relation $r w=f(r, w)$ is a consequence of $R \cup U$ for all $r \in Q^{1}$ and $w \in\left(\left(A \cup A^{-1}\right) \cup\left(Q \cup Q^{-1}\right)\right)^{*}$ such that $(r, w) \neq(1,1)$. 
Proof. The proof is by induction on the length of $w$. If $|w| \leq 1$, then $r w=f(r, w)$ is a relation in $U$ and so we are done. Assume now that $|w| \geq 2$, and write $r w \equiv r x w^{\prime}$, where $x \in\left(A \cup A^{-1}\right) \cup\left(Q \cup Q^{-1}\right)$. Then

$$
f(r, w) \equiv \overline{r x} f\left(q_{r x}, w^{\prime}\right) .
$$

By the inductive hypothesis, $f\left(q_{r x}, w^{\prime}\right)=q_{r x} w^{\prime}$ is a consequence of $R \cup U$, so we can deduce that $f(r, w)=\overline{r x} q_{r x} w^{\prime}$. Since $\overline{r x} q_{r x} \equiv f(r, x)$ and $f(r, x)=r x$ is a relation in $U$, the relation $f(r, w)=r x w^{\prime} \equiv r w$ is a consequence of $R \cup U$.

Proof of Theorem 3.2. Clearly all of the relations in the presentation hold in $S$. To complete the proof, take $\alpha, \beta \in\left(\left(A \cup A^{-1}\right) \cup\left(Q \cup Q^{-1}\right)\right)^{+}$such that $\alpha=\beta$ holds in $S$. We want to show that this relation can be deduced from the relations $R \cup U$. By Lemma 3.3, we can deduce that $\alpha=f(1, \alpha) \in N$ and $\beta=f(1, \beta) \in N$ as a consequence of the relations $R \cup U$. Hence, without loss of generality, we may assume that $\alpha$ and $\beta$ in normal form, say $\alpha \equiv w q \in N$ and $\beta \equiv v r \in N$ where $q, r \in Q$ and $v, w \in\left(A \cup A^{-1}\right)^{*}$.

If $q=r$, then by Lemma 3.1 we know that $w q=v q$ in $S$ if and only if $w=v$ holds in $K$. This last relation can be deduced from the relations in $R$. Hence $\alpha=w q=v q=\beta$ is a consequence of $R \cup U$.

Assume now that $q \neq r$. Since $w q=v r$ holds in $S$ and these words are in normal form, we know that $\pi(q)=\pi(w q)=\pi(v r)=\pi(r)$. It follows that $q r^{-1}$ and $r q^{-1}$ represent elements from $K$.

First we show that $w q=w q r^{-1} r$ can be deduced from the relations in $R \cup U$. Using the second set of relations from $U$ we obtain

$$
w q r^{-1} r=w\left(q q^{-1} q\right) r^{-1} r \equiv w q\left(q^{-1} q\right)\left(r^{-1} r\right)=w q r^{-1} r q^{-1} q=w \widehat{q r^{-1}} \widehat{r q^{-1}} q,
$$

which, letting $w^{\prime} \equiv w \widehat{q r^{-1}} \widehat{r q^{-1}} \in\left(A \cup A^{-1}\right)^{*}$, shows that $w q r^{-1} r=w^{\prime} q$ is a consequence of $R \cup U$. It is clear that $w^{\prime} \in\left(A \cup A^{-1}\right)^{*}$, and $\pi\left(w^{\prime} q\right)=\pi(w q)=\pi(q)$. Furthermore,

$$
w^{\prime} q q^{-1}=\widehat{w q r^{-1}} \widehat{r q^{-1}} q q^{-1}=w^{\prime} .
$$

Thus $w^{\prime} q$ is in normal form. We know that $w^{\prime} q=w q$ holds in $S$ and both these words are in normal form. By Lemma 3.1, this implies that $w=w^{\prime}$ holds in $K$. Thus $w=w^{\prime}$ is a consequence of $R$, and it follows that $w^{\prime} q=w q$ is also a consequence of $R$. Thus $w q=w^{\prime} q=w q r^{-1} r$ is a consequence of the relations $R \cup U$.

Then, as a consequence of $R \cup U$,

$$
w q=w q r^{-1} r=w \widehat{q r^{-1}} r \equiv w^{\prime \prime} r,
$$

where $w^{\prime \prime} \equiv w \widehat{q r^{-1}}$, which is in normal form since $\pi\left(w^{\prime \prime} r\right)=\pi(w q)=\pi(r)$. It follows that $w^{\prime \prime} r=v r$ holds in $S$. By Lemma 3.1, $w^{\prime \prime}=v$ holds in $K$ and so it must be a consequence of $R$. We conclude that $\alpha \equiv w q=v r \equiv \beta$ is a consequence of $R \cup U$.

\section{A presentation for the kernel}

Let $S$ be an inverse semigroup and suppose that $(S, \pi)$ is an extension of $K$ by $T$. Let $\langle Y \mid R\rangle$ be an inverse semigroup presentation for $S$ and let $A=Y \cup Y^{-1}$. In this 
section, we show how to obtain a presentation for the kernel $K$. The proof is more involved than the other direction, which we dealt with in the previous section. This is not very surprising since, when applied to normal subgroups of groups, this result generalises the well-known Reidemeister-Schreier theorem for groups, which rewrites a presentation for the group to a presentation for the subgroup. After introducing the necessary definitions and notation the presentation for $K$ is then given in Theorem 4.4, and the remainder of the section is devoted to proving that this presentation indeed defines $K$. This is achieved in two stages: first we show how to rewrite a generating set for $S$ into a generating set for $K$, and then we show how to rewrite the defining relations of $S$ to obtain a set of defining relations for $K$.

As in the previous section, Lemma 2.1 will be fundamental for the proof, and the question of preservation of finiteness, both of the generating set and of the presentation, will reduce to the question of whether or not one is able to choose the set $Q$ to be finite. This will be discussed in more detail in Section 5 when we look at finiteness properties.

We use the same notation as in previous sections. Thus, for all $t \in T$, we have $C_{t}=\pi^{-1}(t)$,

$$
E_{t}=\left\{s^{-1} s: s \in C_{t}\right\}
$$

and $F_{t} \subseteq E_{t}$ is a fixed subset with the property that for all $e \in E_{t}$, there exists $f_{e} \in F_{t}$ such that $e \leq f_{e}$. Also, for each $t \in T$ and $f \in F_{t}$, we fix a representative $s_{f, t} \in C_{t}$ such that $f=s_{f, t}^{-1} s_{f, t}$, and then define

$$
Q_{t}=\left\{s_{f, t}: f \in F_{t}\right\} \quad \text { and } \quad Q=\bigcup_{t \in T} Q_{t} .
$$

Abusing notation slightly, in this section we view $Q$ as a subset of $A^{+}=\left(Y \cup Y^{-1}\right)^{+}$ with every $q \in Q$ given by a fixed word in $A^{+}$representing the element $q$. Also, as in the previous section, we extend the mapping $\pi: S \rightarrow T$ to $\pi: A^{+} \rightarrow T$ in the obvious way, and we use the same symbol $\pi$ for both maps.

In order to describe a generating set and presentation for $K$ we shall need the following definition. Define a right action of the free monoid $A^{*}$ on the set of words $Q^{1}=Q \cup\{1\}$ (the set $Q$ together with the empty word 1) in the following way. First set $q \cdot 1=q$ for all $q \in Q^{1}$. Then for every $q \in Q^{1}$ and $a \in A$, choose and fix an element $q \cdot a \in Q_{\pi(q a)}$ satisfying

$$
q a=q a(q \cdot a)^{-1}(q \cdot a) .
$$

Such an element exists by Lemma 2.1. Then extend this to an action

$$
Q^{1} \times A^{*} \rightarrow Q^{1}, \quad(q, w) \mapsto q \cdot w,
$$

where we define inductively

$$
q \cdot w a=(q \cdot w) \cdot a,
$$

for $w \in A^{+}$and $a \in A$. The following lemma may be proved by an easy induction on the length of the word $w$, so we omit the proof.

LEMMA 4.1. The mapping

$$
Q^{1} \times A^{*} \rightarrow Q^{1}, \quad(q, w) \mapsto q \cdot w
$$


defines an action of the free monoid $A^{*}$ on the set $Q^{1}$. Moreover, we have the following equalities:

(i) $\quad q w=q w(q \cdot w)^{-1}(q \cdot w)$ in $S$; and

(ii) $\pi(q w)=\pi(q) \pi(w)=\pi(q \cdot w)$,

for all $q \in Q^{1}$ and $w \in A^{*}$ with $(q, w) \neq(1,1)$.

In particular we see that $q a(q \cdot a)^{-1} \in \mathcal{L}(A, K)$ for all $q \in Q^{1}$ and $a \in A$, where $\mathcal{L}(A, K)$ is the set of all words from $A^{*}$ representing elements of $K$. It is important to note that it is not the case here that we have an action of the semigroup $S$ on the set $Q^{1}$, that is, in general two words representing the same element of $S$ can act differently on $Q^{1}$.

The following result is a direct generalisation of Schreier's lemma, which gives generators for a subgroup of a group (see, for instance, [32, Ch. II]).

Proposition 4.2. Let $S$ be an inverse semigroup generated by a set $Y$, and suppose that $(S, \pi)$ is an extension of $K$ by $T$. Then with $A=Y \cup Y^{-1}$, and the notation above, $K$ is generated by the set

$$
X=\left\{q a(q \cdot a)^{-1}: q \in Q^{1}, a \in A\right\} \cup(Q \cap K) .
$$

Proof. Let $w \in K$ be arbitrary. Write $w=a_{1} \cdots a_{n}$ where $a_{1}, a_{2}, \ldots, a_{n} \in A$. Let $r_{i}=1 \cdot a_{1} \cdots a_{i}$ for $i=1, \ldots, n$. Then

$$
\begin{aligned}
w & =1 a_{1} \cdots a_{n} \\
& =\left(1 a_{1} r_{1}^{-1} r_{1}\right) a_{2} \cdots a_{n} \\
& =\left(1 a_{1} r_{1}^{-1}\right)\left(r_{1} a_{2}\right) a_{3} \cdots a_{n} \\
& =\left(1 a_{1} r_{1}^{-1}\right)\left(r_{1} a_{2} r_{2}^{-1} r_{2}\right) a_{3} \cdots a_{n} \\
& =\left(1 a_{1} r_{1}^{-1}\right)\left(r_{1} a_{2} r_{2}^{-1}\right)\left(r_{2} a_{3}\right) a_{4} \cdots a_{n} \\
& =\cdots \\
& =\left(1 a_{1} r_{1}^{-1}\right)\left(r_{1} a_{2} r_{2}^{-1}\right) \cdots\left(r_{n-1} a_{n} r_{n}^{-1}\right) r_{n},
\end{aligned}
$$

where $1 a_{1} r_{1}^{-1}=1 a_{1}\left(1 \cdot a_{1}\right)^{-1} \in X$, and for $i=1, \ldots n-1$

$$
r_{i} a_{i+1} r_{i+1}^{-1}=r_{i} a_{i+1}\left(r_{i} \cdot a_{i+1}\right)^{-1} \in X .
$$

By Lemma 4.1, $\pi\left(r_{n}\right)=\pi(1 \cdot w)=\pi(w)$ and hence $r_{n} \in X$. Thus we have expressed $w$ as a product of elements from $X$, and since $w \in K$ was arbitrary, this completes the proof of the proposition.

We now introduce a new alphabet with respect to which we shall define a presentation for $K$. Let

$$
B=\left\{b_{r, a, s^{-1}}: r, s \in Q^{1}, a \in A^{1}, \operatorname{ras}^{-1} \in \mathcal{L}(A, K)\right\} \cup\left\{e_{r, s}: r, s \in Q\right\} .
$$

Note that we allow $r=1$ or $s=1$ in the expression above, and in these cases both 1 and $1^{-1}$ should be read as the empty word. To simplify the exposition in what follows, 
the generating set $B$ has been chosen to contain some redundant generators (compare with the generating set $X$ for $K$ given in Proposition 4.2).

To simplify notation, for $q \in Q^{1}$ and $a \in A^{1}$ we shall use $b_{q, a}$ as shorthand for the letter $b_{q, a,(q \cdot a)^{-1}}$. Observe that $b_{1,1}=b_{1,1,1^{-1}}=b_{1,1,1} \notin B$ since $1 \notin \mathcal{L}(A, K)$.

Let $\psi: B^{+} \rightarrow A^{+}$be the homomorphism extending

$$
\begin{gathered}
b_{r, a, s^{-1}} \mapsto r a s^{-1} \quad \forall r, s \in Q^{1}, a \in A^{1}, \operatorname{ras}^{-1} \in \mathcal{L}(A, K), \\
e_{r, s} \mapsto r s^{-1} s r^{-1} \quad \forall r, s \in Q .
\end{gathered}
$$

The intended meaning here is that the mapping $\psi$ identifies the word in $A^{+}$that the corresponding letter from $B$ represents.

Next we define a mapping that records the effect of rewriting a word $w \in A^{*}$ by pushing a representative $q$ through from left to right. We define

$$
\phi^{\prime}:\left\{(q, w) \in Q^{1} \times A^{*}: q w \in \mathcal{L}(A, K)\right\} \rightarrow B^{+}
$$

inductively by

$$
\phi^{\prime}(q, w)= \begin{cases}b_{q, 1,1} & \text { if } w=1, \\ b_{q, a} \phi^{\prime}(q \cdot a, u) & \text { if } w \equiv a u \text { where } a \in A \text { and } u \in A^{*} .\end{cases}
$$

Here $\phi^{\prime}(q \cdot a, u)$ is well defined as $\pi((q \cdot a) u)=\pi(q a u)=\pi(q w) \in E(T)$ by Lemma 4.1, that is, $(q \cdot a) u \in \mathcal{L}(A, K)$. Note that $(1,1)$ is not in the domain of $\phi^{\prime}$ since $1 \notin \mathcal{L}(A, K)$. Also define the mapping

$$
\phi: \mathcal{L}(A, K) \rightarrow B^{+}, \quad \phi(w)=\phi^{\prime}(1, w) .
$$

To simplify notation slightly, for $w \equiv a_{1} \cdots a_{n} \in A^{*}$ and $q_{0} \in Q^{1}$, we define

$$
\beta_{q_{0}, w} \equiv b_{q_{0}, a_{1}} b_{q_{1}, a_{2}} b_{q_{2}, a_{3}} \cdots b_{q_{n-1}, a_{n}} \in B^{*}
$$

where $q_{i} \equiv q_{0} \cdot a_{1} a_{2} \cdots a_{i}$ for $1 \leq i \leq n$. Note that in particular $\beta_{1,1}$ and $\beta_{q, 1}(q \in Q)$ are all empty products. In other words $\beta_{1,1} \equiv \beta_{q, 1} \equiv 1 \in B^{*}$ for all $q \in Q$. The following easy lemma describes the effect of applying the mapping $\phi^{\prime}$. We omit the proof since it is a simple consequence of the definitions.

Lemma 4.3. Let $q \in Q^{1}$ and $w \equiv a_{1} \cdots a_{k} \in A^{*}$ with $q w \in \mathcal{L}(A, K)$. Then, setting $q_{i}=$ $q \cdot a_{1} \cdots a_{i}$

$$
\phi^{\prime}(q, w) \equiv b_{q, a_{1}} b_{q_{1}, a_{2}} b_{q_{2}, a_{3}} \cdots b_{q_{k-1}, a_{k}} b_{q_{k}, 1,1} \equiv \beta_{q, w} b_{q_{k}, 1,1} .
$$

It follows that, for all $q \in Q^{1}$ and $u, v \in A^{*}$ such that $q u v \in \mathcal{L}(A, K)$,

$$
\phi^{\prime}(q, u v) \equiv \beta_{q, u} \phi^{\prime}(q \cdot u, v) .
$$

Note that $b_{q, 1,1} \in B$ and $\psi\left(b_{q, 1,1}\right)=q \in A^{*}$ for all $q \in Q \cap \mathcal{L}(A, K)$.

We are now in a position to state the main result of this section, which shows how one may obtain a presentation for $K$ from a presentation for $S$. We note that in the presentation $\langle B \mid \mathcal{D}\rangle$ for $K$ obtained in the following theorem, the set $B$ is an abstract alphabet and is not a subset of $K$. The element of $K$ that a given letter of $B$ represents 
may be obtained by applying the mapping $\psi$ to the letter to obtain a word in $A^{+}$, and then taking the element in $K$ that this word represents (see the definition of $\psi$ above).

THeORem 4.4. Let $S$ be an inverse semigroup defined by an inverse semigroup presentation $\langle Y \mid R\rangle$, and suppose that $(S, \pi)$ is an extension of $K$ by $T$. Then with

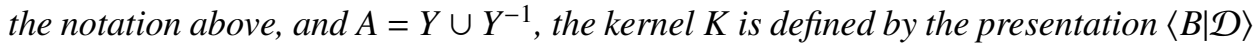
where

$$
B=\left\{b_{r, a, s^{-1}}: r, s \in Q^{1}, a \in A^{1}, \operatorname{ras}^{-1} \in \mathcal{L}(A, K)\right\} \cup\left\{e_{r, s}: r, s \in Q\right\},
$$

and $\mathcal{D}$ consists of the relations (4.1)-(4.10) below, where $a_{i} \in A, r_{i}, s_{i} \in Q$, and $s_{i+1}=s_{i} \cdot a_{i+1}$ and $r_{i+1}=r_{i} \cdot a_{i+1}$, and for all $i$,

$$
\begin{aligned}
\phi\left(\text { ras }^{-1}\right) & =b_{r, a, s^{-1}} \quad \forall r, s \in Q^{1}, a \in A^{1}, \operatorname{ras}^{-1} \in \mathcal{L}(A, K), \\
b_{r, 1,1} b_{1, a} & =b_{r, a,(1 \cdot a)^{-1}} \quad \forall r \in Q \cap \mathcal{L}(A, K), a \in A, \\
b_{r_{1}, a_{2}, s_{2}^{-1}} b_{s_{2}, a_{3}} & =b_{r_{1}, a_{2}} e_{r_{2}, s_{2}} b_{r_{2}, a_{3}, s_{3}^{-1}} \quad \forall r_{2} a_{3} s_{3}^{-1}, r_{1} a_{2} s_{2}^{-1} \in \mathcal{L}(A, K), \\
b_{r_{1}, a_{2}, s_{2}^{-1}} b_{s_{2}, 1,1} & =b_{r_{1}, a_{2}} e_{r_{2}, s_{2}} b_{r_{2}, 1,1} \quad \forall r_{2}, s_{2} \in \mathcal{L}(A, K), r_{1} a_{2} s_{2}^{-1} \in \mathcal{L}(A, K), \\
e_{r_{1}, s_{1}} b_{r_{1}, a_{2}} & =e_{r_{1}, s_{1}} b_{r_{1}, a_{2}} e_{r_{2}, s_{2}}, \\
b_{r_{0}, a_{1}} & =b_{r_{0}, a_{1}} e_{r_{1}, s_{1}} \quad \text { where } s_{1}=1 \cdot a_{1},
\end{aligned}
$$

and with $(u=v) \in R, q_{0} \in Q^{1}, s_{0}=q_{0} \cdot u, t_{0}=q_{0} \cdot v$ and $t_{i+1}=t_{i} \cdot a_{i+1}$ for all $i$

$$
\begin{aligned}
\beta_{q_{0}, u} b_{s_{0}, a_{1}, s_{1}^{-1}} & =\beta_{q_{0}, v} b_{t_{0}, a_{1}, s_{1}^{-1}}, \\
\beta_{q_{0}, u} b_{s_{0}, 1,1} & =\beta_{q_{0}, v} b_{t_{0}, 1,1}, \\
\beta_{q_{0}, v} b_{t_{0}, a_{1}} e_{t_{1}, s_{1}} b_{t_{1}, a_{2}} & =\beta_{q_{0}, v} b_{t_{0}, a_{1}} b_{t_{1}, a_{2}}, \\
\beta_{q_{0}, v} b_{t_{0}, a_{1}} e_{t_{1}, s_{1}} b_{t_{1}, 1,1} & =\beta_{q_{0}, v} b_{t_{0}, a_{1}} b_{t_{1}, 1,1} .
\end{aligned}
$$

In particular, if $S$ is finitely presented, and $Q$ is finite, then $K$ is finitely presented.

The rest of this section will be devoted to the proof of Theorem 4.4. We note that, in general, finiteness of $T$ is not enough to guarantee finiteness of the presentation above, and in this aspect the result above cannot be improved; see the example in Proposition 5.2 below. However, there are many natural situations where $Q$ is finite, and hence the presentation above will be finite. For instance, clearly when the result is applied to groups the set $Q$ is automatically finite, since a group has only finitely many idempotents, and thus the classical Reidemeister-Schreier theorem for groups (in the case of passing to normal subgroups) may be recovered as a special case of the result above.

The proof of Theorem 4.4 has two parts: first we show all the relations hold in $K$, meaning that the relation holds in $K$ once the map $\psi$ has been applied, and then that every relation in $K$ may be deduced from these relations.

4.1. The relations $\mathcal{D}$ all hold. We begin by showing that each of the relations in the presentation holds in $K$. This means that for every relation $u=v$ from $\mathcal{D}$ we 
must check that the relation $\psi(u)=\psi(v)$ holds in $S$. We must also verify that the letters appearing in the relations really do all belong to $B$, that is, for each letter $b_{r, a, s^{-1}}$ appearing in the presentation we must check that $\operatorname{ras}^{-1} \in \mathcal{L}(A, K)$.

We begin with two easy lemmas.

Lemma 4.5. Let $q \in Q$ and $w \equiv a_{1} \cdots a_{k} \in A^{*}$ with $q w \in \mathcal{L}(A, K)$. Then

$$
\psi\left(\phi^{\prime}\left(q, a_{1} \cdots a_{k}\right)\right) \equiv q a_{1} q_{1}^{-1} q_{1} a_{2} q_{2}^{-1} q_{2} a_{3} \cdots a_{k} q_{k}^{-1} q_{k},
$$

where $q_{i}=q \cdot a_{1} \cdots a_{i}$ for $1 \leq i \leq k$. In particular, the relation

$$
\psi\left(\phi^{\prime}(q, w)\right)=q w
$$

holds in $S$.

Proof. The first part is a direct consequence of Lemma 4.3 and the definitions, while the second part follows since

$$
q a_{1} q_{1}^{-1} q_{1} a_{2} q_{2}^{-1} q_{2} a_{3} \cdots a_{k} q_{k}^{-1} q_{k}=q a_{1} a_{2} \cdots a_{k}
$$

in $S$.

Lemma 4.6. Let $w_{1}, w_{2} \in A^{*}$ and $q \in Q$. If $w_{1}=w_{2}$ in $S$, and $q w_{1} \in \mathcal{L}(A, K)$, and hence also $q w_{2} \in \mathcal{L}(A, K)$, then $\psi \phi^{\prime}\left(q, w_{1}\right)=\psi \phi^{\prime}\left(q, w_{2}\right)$ in $S$.

Proof. This is straightforward, since applying Lemma 4.5 we see that in $S$

$$
\psi \phi^{\prime}\left(q, w_{1}\right)=q w_{1}=q w_{2}=\psi \phi^{\prime}\left(q, w_{2}\right)
$$

as required.

We shall now work through the relations one family at a time, checking that they each hold in $K$. The relations (4.1) clearly hold as a consequence of Lemma 4.5.

Claim. Relations (4.2) hold in $K$.

As $r \in \mathcal{L}(A, K)$, it follows that $b_{r, 1,1}, b_{r, a,(1 \cdot a)^{-1}} \in B$. The relation holds in $K$ since

$$
\psi\left(b_{r, 1,1} b_{1, a}\right)=r a(1 \cdot a)^{-1}=\psi\left(b_{r, a,(1 \cdot a)^{-1}}\right)
$$

in $S$, and the claim is proved.

Claim. Relations (4.3) hold in $K$.

Since $r_{2} a_{3} s_{3}^{-1}, r_{1} a_{2} s_{2}^{-1} \in \mathcal{L}(A, K)$ it follows that the letters appearing in relation (4.3) each belong to $B$. Since idempotents commute, and $r_{1} a_{2} r_{2}^{-1} r_{2}=r_{1} a_{2}$ in $S$, by applying $\psi$ we deduce that

$$
\begin{aligned}
\psi\left(b_{r_{1}, a_{2}} e_{r_{2}, s_{2}} b_{r_{2}, a_{3}, s_{3}^{-1}}\right) & =\left(r_{1} a_{2} r_{2}^{-1}\right)\left(r_{2} s_{2}^{-1} s_{2} r_{2}^{-1}\right)\left(r_{2} a_{3} s_{3}^{-1}\right) \\
& =r_{1} a_{2} r_{2}^{-1} r_{2} r_{2}^{-1} r_{2} s_{2}^{-1} s_{2} a_{3} s_{3}^{-1} \\
& =r_{1} a_{2} r_{2}^{-1} r_{2} s_{2}^{-1} s_{2} a_{3} s_{3}^{-1} \\
& =r_{1} a_{2} s_{2}^{-1} s_{2} a_{3} s_{3}^{-1} \\
& =\psi\left(b_{r_{1}, a_{2}, s_{2}^{-1}} b_{s_{2}, a_{3}}\right)
\end{aligned}
$$

and we are done. 
Claim. Relations (4.4) hold in $K$.

Since $r_{2}, s_{2}, r_{1} a_{2} s_{2}^{-1} \in \mathcal{L}(A, K)$ it follows that the letters appearing in the relation come from the set $B$. By applying $\psi$, we obtain

$$
\begin{aligned}
\psi\left(b_{r_{1}, a_{2}} e_{r_{2}, s_{2}} b_{r_{2}, 1,1}\right) & =\left(r_{1} a_{2} r_{2}^{-1}\right)\left(r_{2} s_{2}^{-1} s_{2} r_{2}^{-1}\right)\left(r_{2}\right) \\
& =r_{1} a_{2} r_{2}^{-1} r_{2} r_{2}^{-1} r_{2} s_{2}^{-1} s_{2} \\
& =\left(r_{1} a_{2} r_{2}^{-1} r_{2}\right) s_{2}^{-1} s_{2} \\
& =r_{1} a_{2} s_{2}^{-1} s_{2} \\
& =\psi\left(b_{r_{1}, a_{2}, s_{2}^{-1}} b_{s_{2}, 1,1}\right),
\end{aligned}
$$

as required.

Claim. Relations (4.5) hold in $K$.

The letters in the relation clearly all belong to $B$. Since idempotents commute and $s_{1} a_{2}\left(s_{2}^{-1} s_{2}\right)=s_{1} a_{2}$ in $S$, by applying $\psi$, we obtain

$$
\begin{aligned}
\psi\left(e_{r_{1}, s_{1}} b_{r_{1}, a_{2}} e_{r_{2}, s_{2}}\right) & =\left(r_{1} s_{1}^{-1} s_{1} r_{1}^{-1}\right)\left(r_{1} a_{2} r_{2}^{-1}\right)\left(r_{2} s_{2}^{-1} s_{2} r_{2}^{-1}\right) \\
& =r_{1}\left(s_{1}^{-1} s_{1}\right)\left(r_{1}^{-1} r_{1}\right) a_{2}\left(r_{2}^{-1} r_{2}\right)\left(s_{2}^{-1} s_{2}\right) r_{2}^{-1} \\
& =r_{1}\left(r_{1}^{-1} r_{1}\right)\left(s_{1}^{-1} s_{1}\right) a_{2}\left(s_{2}^{-1} s_{2}\right)\left(r_{2}^{-1} r_{2}\right) r_{2}^{-1} \\
& =r_{1}\left(r_{1}^{-1} r_{1}\right)\left(s_{1}^{-1} s_{1}\right) a_{2}\left(r_{2}^{-1} r_{2}\right) r_{2}^{-1} \\
& =\left(r_{1} s_{1}^{-1} s_{1} r_{1}^{-1}\right)\left(r_{1} a_{2} r_{2}^{-1}\right) \\
& =\psi\left(e_{r_{1}, s_{1}} b_{r_{1}, a_{2}}\right) .
\end{aligned}
$$

(Here in the third line we have underlined the subword to which the relation is being applied for that step.) It follows that relation (4.5) holds in $K$.

Claim. Relations (4.6) hold in $K$.

Clearly each of the letters in the relation belongs to $B$. Since $s_{1}=1 \cdot a_{1}$, idempotents commute and $a_{1} s_{1}^{-1} s_{1}=a_{1}$, applying $\psi$ gives

$$
\begin{aligned}
\psi\left(b_{r_{0}, a_{1}} e_{r_{1}, s_{1}}\right) & =\left(r_{0} a_{1} r_{1}^{-1}\right)\left(r_{1} s_{1}^{-1} s_{1} r_{1}^{-1}\right) \\
& =r_{0} a_{1}\left(r_{1}^{-1} r_{1}\right)\left(s_{1}^{-1} s_{1}\right) r_{1}^{-1} \\
& =r_{0} a_{1}\left(s_{1}^{-1} s_{1}\right)\left(r_{1}^{-1} r_{1}\right) r_{1}^{-1} \\
& =r_{0}\left(a_{1} s_{1}^{-1} s_{1}\right) r_{1}^{-1} \\
& =r_{0} a_{1} r_{1}^{-1} \\
& =\psi\left(b_{r_{0}, a_{1}}\right)
\end{aligned}
$$

as required.

Claim. Relations (4.7) and (4.8) hold in $K$.

We give a proof for the family of relations (4.7), the fact that the relations (4.8) hold in $K$ may be proved similarly. 
Clearly $b_{s_{0}, a_{1}, s_{1}^{-1}} \in B$. Also, since, by Lemma 4.1,

$$
\pi\left(s_{0}\right)=\pi\left(q_{0} u\right)=\pi\left(q_{0} v\right)=\pi\left(t_{0}\right),
$$

and thus

$$
\pi\left(t_{0} a_{1} s_{1}^{-1}\right)=\pi\left(s_{0} a_{1} s_{1}^{-1}\right) \in E(T),
$$

it follows that $b_{t_{0}, a_{1}, s_{1}^{-1}} \in B$. By Lemmas 4.3 and 4.5, applying $\psi$ gives

$$
\psi\left(\beta_{q_{0}, u} b_{s_{0}, a_{1}, s_{1}^{-1}}\right)=q_{0} u a_{1} s_{1}^{-1}=q_{0} v a_{1} s_{1}^{-1}=\psi\left(\beta_{q_{0}, v} b_{t_{0}, a_{1}, s_{1}^{-1}}\right),
$$

in $S$, as claimed.

Claim. Relations (4.9) and (4.10) hold in $K$.

We treat the family of relations (4.9); the relations (4.10) may be handled similarly.

Clearly all the letters appearing in this relation belong to $B$. First observe that

$$
\psi\left(\beta_{q_{0}, v} b_{t_{0}, a_{1}} e_{t_{1}, s_{1}} b_{t_{1}, a_{2}}\right) \equiv \psi\left(\beta_{q_{0}, v}\right)\left(t_{0} a_{1} t_{1}^{-1}\right)\left(t_{1} s_{1}^{-1} s_{1} t_{1}^{-1}\right)\left(t_{1} a_{2} t_{2}^{-1}\right) .
$$

Since $u=v$, in $S$ we have (again using Lemmas 4.3 and 4.5)

$$
\psi\left(\beta_{q_{0}, v}\right) t_{0} a_{1}=q_{0} v a_{1}=q_{0} u a_{1}
$$

and by Lemma 4.1 ,

$$
\left(q_{0} u a_{1}\right) s_{1}^{-1} s_{1}=\left(q_{0} u a_{1}\right)
$$

It follows that

$$
\psi\left(\beta_{q_{0}, v}\right) t_{0} a_{1}=q_{0} u a_{1}=\left(q_{0} u a_{1}\right) s_{1}^{-1} s_{1}=\left(\psi\left(\beta_{q_{0}, v}\right) t_{0} a_{1}\right) s_{1}^{-1} s_{1},
$$

and therefore in $S$

$$
\begin{aligned}
\psi\left(\beta_{q_{0}, v} b_{t_{0}, a_{1}} e_{t_{1}, s_{1}} b_{t_{1}, a_{2}}\right) & =\psi\left(\beta_{q_{0}, v}\right)\left(t_{0} a_{1} t_{1}^{-1}\right)\left(t_{1} s_{1}^{-1} s_{1} t_{1}^{-1}\right)\left(t_{1} a_{2} t_{2}^{-1}\right) \\
& =\frac{\psi\left(\beta_{q_{0}, v}\right) t_{0} a_{1}\left(s_{1}^{-1} s_{1}\right)\left(t_{1}^{-1} t_{1}\right)\left(t_{1}^{-1} t_{1}\right) a_{2} t_{2}^{-1}}{\psi\left(\beta_{q_{0}, v}\right) t_{0} a_{1}\left(t_{1}^{-1} t_{1}\right)\left(t_{1}^{-1} t_{1}\right) a_{2} t_{2}^{-1}} \\
& =\psi\left(\beta_{q_{0}, v}\right)\left(t_{0} a_{1} t_{1}^{-1}\right)\left(t_{1} a_{2} t_{2}^{-1}\right) \\
& =\psi\left(\beta_{q_{0}, v} b_{t_{0}, a_{1}} b_{t_{1}, a_{2}}\right),
\end{aligned}
$$

as claimed.

This completes the proof that every relation in the presentation $\langle B \mid \mathcal{D}\rangle$ holds in $K$.

4.2. The relations $\mathcal{D}$ suffice. To complete the proof of Theorem 4 .4, we must now show that an arbitrary relation in $K$ can be deduced by the set of relations $\mathcal{D}$ given in the statement of the theorem. In [16], the authors give a procedure to write a presentation for a subsemigroup from a presentation of a semigroup. This method admits a natural and straightforward generalisation to inverse semigroups and inverse semigroup presentations; see [17] for details. It is a consequence of these general results that, with the notation above, the following presentation, which we denote by $\mathcal{P}$, 
defines the kernel $K$ : the generating set is $B$, and the relations are

$$
\begin{aligned}
\phi\left(r a s^{-1}\right) & =b_{r, a, s^{-1}} \quad \forall r, s \in Q^{1}, a \in A^{1}, \operatorname{ras}^{-1} \in \mathcal{L}(A, K), \\
\phi\left(w_{1} w_{2}\right) & =\phi\left(w_{1}\right) \phi\left(w_{2}\right) \quad \forall w_{1}, w_{2} \in \mathcal{L}(A, K), \\
\phi\left(w_{3} u w_{4}\right) & =\phi\left(w_{3} v w_{4}\right) \quad \forall w_{3}, w_{4} \in A^{*},(u=v) \in R, w_{3} u w_{4} \in \mathcal{L}(A, K) .
\end{aligned}
$$

To complete the proof of Theorem 4.4, it will suffice to show that the relations in the presentation $\mathcal{P}$ are all consequences of the set of relations $\mathcal{D}$.

The first family of relations of $\mathcal{P}$ is included in $\mathcal{D}$ and hence automatically deducible. The following two lemmas show how to deal with the remaining two families of relations.

Lemma 4.7. The relations $\phi\left(w_{1} w_{2}\right)=\phi\left(w_{1}\right) \phi\left(w_{2}\right)$, where $w_{1}, w_{2} \in \mathcal{L}(A, K)$, are consequences of the relations in $\mathcal{D}$.

Proof. First write

$$
w_{2} \equiv a_{1} \cdots a_{n}
$$

where $a_{1}, \ldots, a_{n} \in A$. Let $r_{0}=1 \cdot w_{1}$, and, when $1 \leq i \leq n$, define

$$
s_{i}=1 \cdot a_{1} \cdots a_{i}, \quad r_{i}=r_{0} \cdot a_{1} \cdots a_{i} .
$$

By Lemma 4.3, $\phi\left(w_{1}\right)$ decomposes:

$$
\phi\left(w_{1}\right) \equiv \beta_{1, w_{1}} b_{r_{0}, 1,1} .
$$

Applying the last clause of Lemma 4.3, we then have

$$
\phi\left(w_{1}\right) \phi\left(w_{2}\right) \equiv \beta_{1, w_{1}} b_{r_{0}, 1,1} \phi^{\prime}\left(1, w_{2}\right), \quad \phi\left(w_{1} w_{2}\right) \equiv \beta_{1, w_{1}} \phi^{\prime}\left(r_{0}, w_{2}\right) .
$$

We shall now prove that

$$
b_{r_{0}, 1,1} \phi^{\prime}\left(1, w_{2}\right)=\phi^{\prime}\left(r_{0}, w_{2}\right)
$$

is a consequence of the set of relations $\mathcal{D}$. Evaluating the left-hand side of (4.11) we have

$$
b_{r_{0}, 1,1} \phi^{\prime}\left(1, w_{2}\right) \equiv b_{r_{0}, 1,1} b_{1, a_{1}} b_{s_{1}, a_{2}} \cdots b_{s_{n-1}, a_{n}} b_{s_{n}, 1,1},
$$

while from the right-hand side we obtain

$$
\phi^{\prime}\left(r_{0}, w_{2}\right) \equiv b_{r_{0}, a_{1}} b_{r_{1}, a_{2}} b_{r_{2}, a_{3}} \cdots b_{r_{n-1}, a_{n}} b_{r_{n}, 1,1} .
$$

Starting with the left-hand side, first apply (4.2) to obtain

$$
\underline{b_{r_{0}, 1,1} b_{1, a_{1}}} b_{s_{1}, a_{2}} b_{s_{2}, a_{3}} \cdots b_{s_{n-1}, a_{n}} b_{s_{n}, 1,1}=b_{r_{0}, a_{1}, s_{1}^{-1}} b_{s_{1}, a_{2}} b_{s_{2}, a_{3}} \cdots b_{s_{n-1}, a_{n}} b_{s_{n}, 1,1} .
$$

This is an application of a relation from (4.2) since $r_{0}=1 \cdot w_{1} \in \mathcal{L}(A, K)$, because $w_{1} \in \mathcal{L}(A, K)$. Then we continue by applying relations (4.3) successively from left to right:

$$
\begin{aligned}
& \frac{b_{r_{0}, a_{1}, s_{1}^{-1}} b_{s_{1}, a_{2}}}{b_{s_{2}, a_{3}}} b_{s_{3}, a_{4}} \cdots b_{s_{n-1}, a_{n}} b_{s_{n}, 1,1} \\
& \quad=b_{r_{0}, a_{1}} e_{r_{1}, s_{1}} b_{r_{1}, a_{2}, s_{2}^{-1}} b_{s_{2}, a_{3}} b_{s_{3}, a_{4}} \cdots b_{s_{n-1}, a_{n}} b_{s_{n}, 1,1}
\end{aligned}
$$




$$
\begin{aligned}
& =b_{r_{0}, a_{1}} e_{r_{1}, s_{1}} b_{r_{1}, a_{2}} e_{r_{2}, s_{2}} b_{r_{2}, a_{3}, s_{3}^{-1}} b_{s_{3}, a_{4}} \cdots b_{s_{n-1}, a_{n}} b_{s_{n}, 1,1} \\
& =\cdots \\
& =b_{r_{0}, a_{1}} e_{r_{1}, s_{1}} b_{r_{1}, a_{2}} e_{r_{2}, s_{2}} b_{r_{2}, a_{3}} e_{r_{3}, s_{3}} \cdots e_{r_{n-1}, s_{n-1}} b_{r_{n-1}, a_{n}, s_{n}^{-1}} b_{s_{n}, 1,1} .
\end{aligned}
$$

These are each valid applications of a relation from (4.3) since

$$
\pi\left(r_{m-1} a_{m} s_{m}^{-1}\right)=\pi\left(w_{1}\left(a_{1} \cdots a_{m-1} a_{m}\right)\left(a_{1} \cdots a_{m}\right)^{-1}\right) \in E(T)
$$

when $m=1, \ldots, n$ by Lemma 4.1, which implies that $r_{m-1} a_{m} s_{m}^{-1} \in \mathcal{L}(A, K)$. Then apply relation (4.4) to obtain

$$
\begin{aligned}
& b_{r_{0}, a_{1}} e_{r_{1}, s_{1}} b_{r_{1}, a_{2}} e_{r_{2}, s_{2}} b_{r_{2}, a_{3}} e_{r_{3}, s_{3}} \cdots e_{r_{n-1}, s_{n-1}} \frac{b_{r_{n-1}, a_{n}, s_{n}^{-1}} b_{s_{n}, 1,1}}{\quad} \\
& =b_{r_{0}, a_{1}} e_{r_{1}, s_{1}} b_{r_{1}, a_{2}} e_{r_{2}, s_{2}} b_{r_{2}, a_{3}} e_{r_{3}, s_{3}} \cdots e_{r_{n-1}, s_{n-1}} b_{r_{n-1}, a_{n}} e_{r_{n}, s_{n}} b_{r_{n}, 1,1} .
\end{aligned}
$$

The fact that this is a relation from (4.4) follows since $r_{n}, s_{n} \in \mathcal{L}(A, K)$, as

$$
\pi\left(r_{n}\right)=\pi\left(r_{0} \cdot w_{2}\right)=\pi\left(r_{0} w_{2}\right)=\pi\left(w_{1} w_{2}\right) \in E(T)
$$

and

$$
\pi\left(s_{n}\right)=\pi\left(w_{2}\right) \in E(T),
$$

and, as above, $r_{n-1} a_{n} s_{n}^{-1} \in \mathcal{L}(A, K)$. In this way, using the relations (4.2)-(4.4) we have transformed the left-hand side into

$$
b_{r_{0}, a_{1}} e_{1} b_{r_{1}, a_{2}} e_{2} b_{r_{2}, a_{3}} e_{3} \cdots e_{n-1} b_{r_{n-1}, a_{n}} e_{n} b_{r_{n}, 1,1}
$$

where $e_{m}=e_{r_{m}, s_{m}}$, for $m=1, \ldots, n$. To finish the proof, we must apply relations to remove these idempotents from the word (4.12).

Note that relation (4.5) says $e_{i} b_{r_{i}, a_{i+1}}=e_{i} b_{r_{i}, a_{i+1}} e_{i+1}$. So working from right to left, we may remove $e_{n}$, then $e_{n-1}$, right down to $e_{2}$, transforming (4.12) into

$$
b_{r_{0}, a_{1}} e_{1} b_{r_{1}, a_{2}} b_{r_{2}, a_{3}} b_{r_{3}, a_{4}} \cdots b_{r_{n-1}, a_{n}} b_{r_{n}, 1,1} \text {. }
$$

Finally, since $s_{1}=1 \cdot a_{1}$, we may apply the relation

$$
b_{r_{0}, a_{1}} e_{1}=b_{r_{0}, a_{1}}
$$

from (4.6) to obtain

$$
b_{r_{0}, a_{1}} b_{r_{1}, a_{2}} b_{r_{2}, a_{3}} b_{r_{3}, a_{4}} \cdots b_{r_{n-1}, a_{n}} b_{r_{n}, 1,1} .
$$

This shows that using only the relations (4.2)-(4.6) we can deduce the relation (4.11), and thus the relation $\phi\left(w_{1} w_{2}\right)=\phi\left(w_{1}\right) \phi\left(w_{2}\right)$. This completes the proof.

Lemma 4.8. The relations $\phi\left(w_{3} u w_{4}\right)=\phi\left(w_{3} v w_{4}\right)$, where $w_{3}, w_{4} \in A^{*},(u=v) \in R$ and $w_{3} u w_{4} \in \mathcal{L}(A, K)$, are consequences of the relations $\mathcal{D}$.

Proof. The approach is similar to that used in the proof of Lemma 4.7. Let

$$
w_{4} \equiv a_{1} \cdots a_{n}
$$


where $a_{1}, \ldots, a_{n} \in A$ (note that it is possible that $w_{4} \equiv 1$ ), and let $q_{0}=1 \cdot w_{3} \in Q^{1}$. Then by the last clause of Lemma 4.3,

$$
\phi\left(w_{3} u w_{4}\right) \equiv \kappa \phi^{\prime}\left(q_{0}, u w_{4}\right), \quad \phi\left(w_{3} v w_{4}\right) \equiv \kappa \phi^{\prime}\left(q_{0}, v w_{4}\right),
$$

where $\kappa \equiv \beta_{1, w_{3}} \in B^{*}$. Therefore to complete the proof of the lemma it suffices to show that

$$
\phi^{\prime}\left(q_{0}, u w_{4}\right)=\phi^{\prime}\left(q_{0}, v w_{4}\right)
$$

is a consequence of the relations $\mathcal{D}$. Let

$$
s_{0}=q_{0} \cdot u \in Q, \quad t_{0}=q_{0} \cdot v \in Q .
$$

Recall that even though $u=v$ in $S$, it is still possible that $q_{0} \cdot u$ and $q_{0} \cdot v$ are not equal to each another. Again applying Lemma 4.3, we obtain

$$
\phi^{\prime}\left(q_{0}, u w_{4}\right) \equiv \beta_{q_{0}, u} \phi^{\prime}\left(s_{0}, w_{4}\right), \quad \phi^{\prime}\left(q_{0}, v w_{4}\right) \equiv \beta_{q_{0}, v} \phi^{\prime}\left(t_{0}, w_{4}\right) .
$$

The left-hand side decomposes:

$$
\beta_{q_{0}, u} \phi^{\prime}\left(s_{0}, w_{4}\right) \equiv \beta_{q_{0}, u} b_{s_{0}, a_{1}} b_{s_{1}, a_{2}} \cdots b_{s_{n-1}, a_{n}} b_{s_{n}, 1,1}
$$

while the right-hand side decomposes:

$$
\beta_{q_{0}, v} \phi^{\prime}\left(t_{0}, w_{4}\right) \equiv \beta_{q_{0}, v} b_{t_{0}, a_{1}} b_{t_{1}, a_{2}} \cdots b_{t_{n-1}, a_{n}} b_{t_{n}, 1,1}
$$

where

$$
s_{i}=s_{0} \cdot a_{1} \cdots a_{i} \in Q, \quad t_{i}=t_{0} \cdot a_{1} \cdots a_{i} \in Q .
$$

We must show that by applying relations from $\mathcal{D}$ we can transform the word (4.14) into the word (4.15).

If $w_{4} \equiv 1$, then (4.14) is the word $\beta_{q_{0}, u} b_{s_{0}, 1,1},(4.15)$ is the word $\beta_{q_{0}, v} b_{t_{0}, 1,1}$, and we are done since $\beta_{q_{0}, u} b_{s_{0}, 1,1}=\beta_{q_{0}, v} b_{t_{0}, 1,1}$ is one of the relations (4.8).

Now suppose that $\left|w_{4}\right| \geq 1$. Applying the relation (4.7), we can transform

$$
\beta_{q_{0}, u} b_{s_{0}, a_{1}, s_{1}^{-1}} b_{s_{1}, a_{2}} b_{s_{2}, a_{3}} \cdots b_{s_{n-1}, a_{n}} b_{s_{n}, 1,1}
$$

into

$$
\beta_{q_{0}, v} b_{t_{0}, a_{1}, s_{1}^{-1}} b_{s_{1}, a_{2}} b_{s_{2}, a_{3}} \cdots b_{s_{n-1}, a_{n}} b_{s_{n}, 1,1} .
$$

Then exactly as in the proof of Lemma 4.7, we apply relations (4.3) and (4.4), working from left to right, to transform (4.16) into

$$
\beta_{q_{0}, v} b_{t_{0}, a_{1}} e_{t_{1}, s_{1}} b_{t_{1}, a_{2}} e_{t_{2}, s_{2}} \cdots e_{t_{n-1}, s_{n-1}} b_{t_{n-1}, a_{n}} e_{t_{n}, s_{n}} b_{t_{n}, 1,1} \text {. }
$$

To see that each of these relations is a valid application of a relation from (4.3) (similarly for (4.4)) one just has to observe that

$$
\pi\left(t_{m} a_{m+1} s_{m+1}^{-1}\right)=\pi\left(q_{0} v a_{1} \cdots a_{m} a_{m+1}\left(q_{0} u a_{1} \cdots a_{m+1}\right)^{-1}\right) \in E(T)
$$

when $m=1, \ldots, n-1$, which implies that $t_{m} a_{m+1} s_{m+1}^{-1} \in \mathcal{L}(A, K)$.

Next, again as in the proof of Lemma 4.7, working from right to left we apply relations (4.5) to remove the idempotents, one at a time. This transforms the word into

$$
\beta_{q_{0}, v} b_{t_{0}, a_{1}} e_{t_{1}, s_{1}} b_{t_{1}, a_{2}} b_{t_{2}, a_{3}} \cdots b_{t_{n-1}, a_{n}} b_{t_{n}, 1,1}
$$


Finally we apply relation (4.9) to transform (4.17) into

$$
\beta_{q_{0}, v} b_{t_{0}, a_{1}} b_{t_{1}, a_{2}} b_{t_{2}, a_{3}} \cdots b_{t_{n-1}, a_{n}} b_{t_{n}, 1,1} .
$$

(In the case that $\left|w_{4}\right|=1$, so $w_{4} \equiv a_{1}$, in this last step we apply the corresponding relation from (4.10) instead here.) In conclusion, we have shown how, using the relations from $\mathcal{D}$, one can deduce the relation (4.13), and hence also the relation $\phi\left(w_{3} u w_{3}\right)=\phi\left(w_{3} v w_{4}\right)$. This completes the proof of the lemma.

This completes the proof of Theorem 4.4.

\section{Applications: finiteness properties}

Let $S$ be an inverse semigroup, and suppose that $(S, \pi)$ is an extension of $K$ by $T$. We now turn our attention to the general question of how the properties of $S, K$ and $T$ are related. As we saw in the Introduction, in the special case that $S$ is a group, there are numerous results relating the finiteness properties of $S, T$ and $K$. In this section, and the next, we shall investigate to what extent results such as these hold for inverse semigroups in general. We begin in this section by considering the situation where $T$ is finite, and we ask about the relationship between properties of $S$ and those of the kernel $K$.

5.1. Some easy properties. For certain finiteness conditions it is easily shown, either by direct proof or by appealing to known results, that, under the assumption that $T$ is finite, $S$ has the property if and only $K$ does. The following theorem summarises a few of these straightforward facts. Recall that a semigroup $S$ is called locally finite if for any finite subset $X$ of $S$ the subsemigroup generated by $X$ is finite, and $S$ is called periodic if for every element $s \in S$ there exist distinct positive integers $i$ and $j$ such that $s^{i}=s^{j}$.

THEOREM 5.1. Let $S$ be an inverse semigroup and suppose that $(S, \pi)$ is an extension of $K$ by $T$. If $T$ is finite, then the following results hold.

(i) The semigroup $S$ is finite if and only if $K$ is finite.

(ii) The semigroup $S$ is periodic if and only if $K$ is periodic.

(iii) The semigroup $S$ is locally finite if and only if $K$ is locally finite.

Proof. The first two parts are easy exercises, while the third follows from a wellknown result of Brown $[13,14]$.

5.2. Finite generation and presentability. As we shall see below in Proposition 5.2, in contrast to the behaviour of the finiteness properties listed in Theorem 5.1, just assuming that $T$ is finite is not strong enough to ensure that either finite generation or presentability are inherited by $K$ from $S$. Therefore an additional assumption is needed. We begin now by presenting a counterexample to the general statement, and then afterwards shall introduce an additional notion, that of being finitely covered, that, once imposed, will yield positive results about preservation of finite generation 
and presentability. This additional hypothesis is still weak enough to allow the result to be applied in many situations, including the case of subgroups of groups.

The following proposition concerns the free inverse monoid $\operatorname{FI}(X)$ on a set $X$. We shall view elements of $\mathrm{FI}(X)$ by means of Munn trees. We give some basic background on Munn trees here. For a more detailed introduction we refer the reader to [28, Section 6.4], or originally [35].

Let $\Gamma$ denote the Cayley graph of the free group $\mathrm{FG}(X)$. Of course, $\Gamma$ is a tree. Given a word $u=x_{1} \cdots x_{n} \in\left(X \cup X^{-1}\right)^{*}$, the Munn tree of $u$ is the finite subtree of $\Gamma$ traversed when the path labelled by the word $u$ is read in $\Gamma$, starting at the vertex 1 and ending at the vertex $r(u)$ (the reduced form of $u$, where reduced here means reduced in the free group). We denote the Munn tree of $u$ by $\operatorname{MT}(u)$, and view it as a birooted labelled subtree of $\Gamma$ with initial vertex 1 and terminal vertex $r(u)$. Munn trees provide a solution to the word problem in free inverse semigroups: two words $u, v$ over $X \cup X^{-1}$ are equal in $\operatorname{FI}(X)$ if and only if $\operatorname{MT}(u)=\operatorname{MT}(v)$. Also $u$ is an idempotent if and only if $r(u)=1$. When drawing Munn trees, we represent the initial vertex (that is, 1) using an inward arrow and the terminal vertex using an outward arrow. In terms of Munn trees, multiplication of elements of $\operatorname{FI}(X)$ is carried out in a natural way, with the product of two trees given by translating the second tree so that its initial vertex coincides with the terminal vertex of the first. Also, for idempotents $e$ and $f$ in the free inverse semigroup, $e \leq f$ if and only if the Munn tree of $e$ can be embedded into the Munn tree of $f$, with the initial vertex of $e$ mapped to the initial vertex of $f$.

Proposition 5.2. Let $S$ denote the free inverse monoid on two generators $x$ and $y$, let $T=\left\{1, a, a^{2}\right\}$ be the cyclic group of order three generated by $a$, let $\pi: S \rightarrow T$ be the unique homomorphism extending $\phi(x)=\phi(y)=a$, and let $K$ denote the kernel of $\pi$. Then $S$ is finitely presented and $T$ is finite, but $K$ is not finitely generated.

Proof. It is clear that the congruence associated with $f$ has three congruence classes, namely $C_{a}, C_{a^{2}}$ and $C_{1}=K=\operatorname{ker}(f)$. Also, it is easy to check that the words $k_{i}=x y\left(x^{-1} y\right)^{i} x^{-1} y^{-1}$ represent elements from $K$ for all $i \geq 1$. The Munn tree of $k_{i}$ is shown in Figure 1. We claim that the element $k_{i}$ cannot be decomposed into a product of elements from $K \backslash\left\{k_{i}\right\}$ for any $i$. Indeed, suppose that we could write $k_{i}=b c$ where $b, c \in K$. Then $\operatorname{MT}(b)$ must be a subtree of $\operatorname{MT}\left(k_{i}\right)$ with the same initial vertex. Note that every vertex in $\operatorname{MT}\left(k_{i}\right)$ other than $\iota$ or $\tau$ corresponds to an element of $C_{a}$ or of $C_{a^{2}}$. Thus the terminal vertex of $\operatorname{MT}(b)$ is either $\tau$ or $\iota$. In the former case, $b=k_{i}$. In the latter case, $\operatorname{MT}(c)$ must start at $\iota$ and end at $\tau$, so that $c=k_{i}$. This proves our claim, and it follows that the set $\left\{k_{i}: i=1,2, \ldots\right\}$ is contained in every generating set of $K$, and so $K$ is not finitely generated.

It follows from Proposition 5.2 that there is no hope of improving the final statement of Theorem 4.4 by obtaining a presentation whose finiteness depends only on that of the original presentation for $S$, and the finiteness of $T$. In other words, the nature of the homomorphism $\pi$, and the relationship with the semilattice of idempotents must also play a role. 


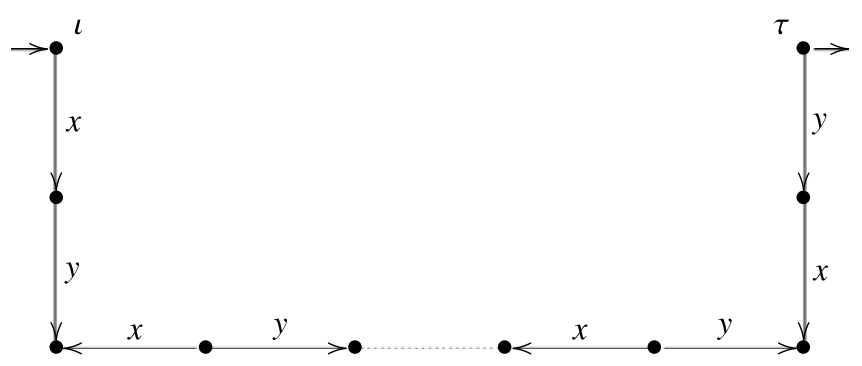

FIgUre 1. The Munn tree of $k_{i}=x y\left(x^{-1} y\right)^{i} x^{-1} y^{-1}$. There are $i$ horizontal $x$ and $y$.

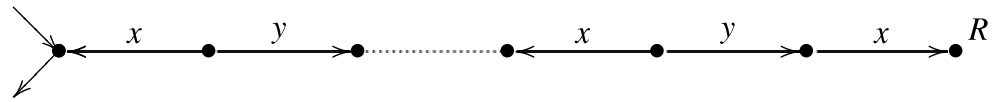

FIGURE 2. The Munn tree of $e_{i}=\left(x^{-1} y\right)^{i} x x^{-1}\left(y^{-1} x\right)^{i}$. There are $i$ horizontal $y$.

Considering again the example in Proposition 5.2 we can see precisely why the set $Q$ cannot be chosen to be finite. Let $n(z, w)$ denote the number of occurrences of the letter $z$ in the word $w$. Let te $(w)$ be the total exponent of the word $w$ in FI, that is,

$$
\text { te }(w)=n(x, w)+n(y, w)-n\left(x^{-1}, w\right)-n\left(y^{-1}, w\right) .
$$

With this notation we can now write the congruence classes associated with the homomorphism $f$ as follows:

$$
C_{a^{i}}=\{w \in \mathrm{FI}: \operatorname{te}(w) \equiv i \bmod 3\} .
$$

It is clear that the idempotents $x^{-1} x$ and $y^{-1} y$ belong to $E_{a}$, and the idempotents $x x^{-1}$ and $y y^{-1}$ belong to $E_{a^{2}}$. (Recall the notation $E_{t}$ from Section 2.) It follows that the idempotents $\left(x^{-1} y\right)^{i} x x^{-1}\left(y^{-1} x\right)^{i}$ also belong to $E_{a^{2}}$ for all $i \geq 1$. The element $e_{i}=\left(x^{-1} y\right)^{i} x x^{-1}\left(y^{-1} x\right)^{i}$ is represented by the Munn tree in Figure 2.

These idempotents are all maximal in $E_{a^{2}}$. Indeed, notice that any vertex of $\operatorname{MT}\left(e_{i}\right)$ other than $R$ corresponds to an element of $C_{a}$ or $C_{1}$. Hence no idempotent larger than $e_{i}$ belongs to $E_{a^{2}}$. In this way we obtain an infinite set of maximal idempotents in the congruence class $E_{a^{2}}$. But $F_{a^{2}}$ is meant to have the property that every $e \in E_{a^{2}}$ lies below some member of $F_{a^{2}}$. This means that $F_{a^{2}}$ cannot be chosen to be finite, and hence the set $Q$ cannot be chosen to be finite.

Remark 5.3. The example in Proposition 5.2 highlights a major difference in behaviour of the free inverse semigroup and the free group, since it is well known (see [33, Theorem 2.10]) that if $\pi: F \rightarrow G$ is a homomorphism from a finitely generated free group $F$ onto a group $G$, then the kernel $K$ of $\pi$ is finitely generated if and only if $G$ is finite (this fact is usually referred to as Greenberg's theorem). Of course, this leaves us with the following natural problem: characterise the finitely generated kernels of free inverse semigroups (the example above shows that finiteness of the image is not enough to ensure finite generation of the kernel). 
Let us now introduce a finiteness condition that guarantees finite generation and presentability of the kernel.

Definition 5.4. Let $(S, \pi)$ be an extension of $K$ by $T$. For $t \in T$ let $C_{t}=\pi^{-1}(t)$ and $E_{t}=\left\{s^{-1} s: s \in C_{t}\right\}$. If, for every $t \in T$, the set of maximal elements $\mathcal{M}\left(E_{t}\right)$ of $E_{t}$ is finite, and every element of $E_{t}$ lies below some element of $\mathcal{M}\left(E_{t}\right)$, we say that the homomorphism $\pi$ is finitely covered.

Clearly if $\pi$ is finitely covered and $T$ is finite, then all the sets $F_{t}$ (where $t \in T$ ) can be chosen to be finite, and hence $Q$ will be finite. Thus, with this notion, as corollaries of Theorems 4.4 and 3.2 we obtain the following result.

THEOREM 5.5. Let $S$ be an inverse semigroup and suppose that $(S, \pi)$ is an extension of $K$ by $T$. If $T$ is finite and $\pi$ is finitely covered, then the following results hold.

(i) The semigroup $S$ is finitely generated if and only if $K$ is finitely generated.

(ii) The semigroup $S$ is finitely presented if and only if $K$ is finitely presented.

Of course, in the case of groups the finitely covered hypothesis is always satisfied (for any homomorphism $\pi$ ) since a group has only finitely many idempotents (indeed, just one). Hence, in particular, Theorem 5.5 has the corresponding group theoretic result as a corollary. However, Theorem 5.5 is more widely applicable. We now list some situations where it can be applied.

Let $S$ be an inverse semigroup and suppose that $(S, \pi)$ is an extension of $K$ by $T$. If any of the following are satisfied, then $\pi$ is finitely covered.

(i) The semigroup $S$ has finitely many idempotents.

(ii) The poset $(E(S), \leq)$ is the reverse of a well-ordering (that is, every subset has a maximal element).

(iii) The homomorphism $\pi$ is an $F$-morphism (in the sense of [29]).

(iv) The homomorphism $\pi$ induces a Billhardt congruence (in the sense of [8] or [28, Section 5.3]).

A natural class of examples of inverse semigroups with property (i) is given by finitely generated inverse subsemigroups of the monoid $M_{n}(K)$ of all $n \times n$ matrices over a field $K$; see [43, Lemma 7.24]. Examples of (ii) include the bicyclic monoid, and, more generally, Bruck-Reilly extensions of groups (see [28, Section 5.4]).

Remark 5.6. The example in Proposition 5.2 shows that if we remove the condition that $\pi$ is finitely covered, then Theorem 5.5 no longer holds. However, it would be of interest to investigate to what extent (if any) the finitely covered hypothesis might be weakened, while still maintaining the result.

Remark 5.7. The example in Proposition 5.2 demonstrates that the finitely covered hypothesis is necessary in order to prove the forward direction of Theorem 5.5(i) and (ii). However, there still remains the question of whether the finitely covered hypothesis is really necessary for the other direction of the theorem. We leave this as an open problem.

5.3. The word problem. Recall that we say an inverse semigroup $S$ with finite generating set $A$ has a soluble word problem (with respect to $A$ ) if there exists an 
algorithm, which, for any two words $u, v \in\left(A \cup A^{-1}\right)^{*}$, decides whether the relation $u=v$ holds in $S$ or not. It is easy to see that solubility of the word problem does not depend on the choice of finite generating set for $S$.

The following result concerning the word problem follows straight from the arguments in the proof of Theorem 3.2. We leave the task of filling in the details as an exercise for the reader.

THEOREM 5.8. Let $S$ be a finitely generated inverse semigroup and suppose that $(S, \pi)$ is an extension of $K$ by $T$. If $T$ is finite, and $\pi$ is finitely covered, then $S$ has a soluble word problem if and only if $K$ has a soluble word problem.

5.4. Residual finiteness. A semigroup $S$ is residually finite if, for any two distinct elements $s, t \in S$, there exists a congruence $\eta$ of finite index (that is, with finitely many congruence classes) that separates these elements, that is, $s \eta \neq t \eta$. The property of residual finiteness in semigroups and inverse semigroups has been considered, for example, in $[4,22]$.

Let $S$ be an inverse semigroup and suppose that $(S, \pi)$ is an extension of $K$ by $T$. All the notation (such as $C_{t}, E_{t}, F_{t}, Q$ ) introduced in Section 2 will remain in force. Moreover, throughout this subsection we will assume that the homomorphism $\pi$ is finitely covered.

We begin with some results concerning the construction and manipulation of congruences on semigroups. Every equivalence relation $\rho$ on the semigroup $S$ gives rise to a right congruence $\Sigma_{r}(\rho)$, the largest right congruence contained in $\rho$ [26, Section 1.5]. Similarly we can define $\Sigma_{l}(\rho)$, the largest left congruence of $S$ contained in $\rho$, and $\Sigma(\rho)$, the largest two-sided congruence of $S$ contained in $\rho$.

The next proposition tells us that any finite index right congruence on $S$ can be refined to give a finite index two-sided congruence.

Proposition 5.9 [39, Theorem 2.4]. Let $\rho$ be a right congruence on $S$. If $\rho$ has finite index, then $\Sigma(\rho)$ has finite index as well.

As a consequence, in order to show that a semigroup $S$ is residually finite, it is sufficient to prove that, whenever $x, y \in S$ and $x \neq y$, there exists a right congruence $\rho$, with finite index, such that $x \rho \neq y \rho$. For any congruence $\eta$ on $K$, we define a relation $\bar{\eta}$ on $S$ as follows: $x \bar{\eta} y$ if and only if $\pi(x)=\pi(y)$ and there exist $p_{1}, \ldots, p_{k} \in S$ and $u_{1}, \ldots, u_{k}, v_{1}, \ldots, v_{k} \in K$ such that

$$
\begin{aligned}
& x=u_{1} p_{1}, \quad \pi(x)=\pi\left(p_{1}\right), \\
& v_{1} p_{1}=u_{2} p_{2}, \quad\left(u_{1}, v_{1}\right) \in \eta, \quad \pi\left(v_{1} p_{1}\right)=\pi\left(p_{2}\right), \\
& v_{2} p_{2}=u_{3} p_{3}, \quad\left(u_{2}, v_{2}\right) \in \eta, \quad \pi\left(v_{2} p_{2}\right)=\pi\left(p_{3}\right), \\
& \cdots, \quad \ldots, \quad \ldots \text {, } \\
& v_{k-1} p_{k-1}=u_{k} p_{k}, \quad\left(u_{k-1}, v_{k-1}\right) \in \eta, \quad \pi\left(v_{k-1} p_{k-1}\right)=\pi\left(p_{k}\right), \\
& v_{k} p_{k}=y, \quad\left(u_{k}, v_{k}\right) \in \eta, \quad \pi\left(v_{k} p_{k}\right)=\pi(y) .
\end{aligned}
$$

LeMma 5.10. The relation $\bar{\eta}$ is a right congruence on $S$. 
Proof. The relation $\bar{\eta}$ is clearly reflexive and symmetric. Transitivity is proved by noting that concatenating two sequences of the form (5.1) yields another sequence of the same form. Likewise, multiplying all the terms in the sequence (5.1) by a fixed element of $S$ yields another sequence of the same form, proving that $\bar{\eta}$ is a right congruence.

Lemma 5.11. If $x, y \in S$ and $x \bar{\eta} y$, then there exists a sequence of the form (5.1) in which every $p_{i}$ belongs to the set $Q$.

Proof. Start with an arbitrary sequence of the form (5.1). Then, applying Lemma 2.1, write $p_{i}=k_{i} q_{i}$ where $k_{i} \in K, q_{i} \in Q$ and $\pi\left(p_{i}\right)=\pi\left(q_{i}\right)$. Then we have $u_{i} p_{i}=\left(u_{i} k_{i}\right) q_{i}$ and $v_{i} p_{i}=\left(v_{i} k_{i}\right) q_{i}$ where $u_{i} k_{i}, v_{i} k_{i} \in K$ and $\left(u_{i} k_{i}, v_{i} k_{i}\right) \in \eta$. Also

$$
\pi\left(v_{i} k_{i} q_{i}\right)=\pi\left(v_{i} p_{i}\right)=\pi\left(p_{i+1}\right)=\pi\left(q_{i+1}\right)
$$

for all $i$. Thus, replacing each $u_{i}$ by $u_{i} k_{i}$, each $v_{i}$ by $v_{i} k_{i}$, and each $p_{i}$ by $q_{i}$ yields a sequence with the desired properties.

Lemma 5.12. If $\eta$ has finite index, then $\bar{\eta}$ has finite index as well.

Proof. Let $R$ be a fixed transversal of the $\eta$-classes of $K$. We claim that the finite set $R Q=\{r q: r \in R, q \in Q\}$ intersects every $\bar{\eta}$-class of $S$. Let $x \in S$ be arbitrary. By Lemma 2.1 write $x=k q$ where $k \in K, q \in Q$ and $\pi(x)=\pi(q)$. Let $r \in R$ be the unique element satisfying $(k, r) \in \eta$, and let $y=r q$. Now

$$
x=k q, \quad r q=y, \quad(k, r) \in \eta
$$

where $\pi(x)=\pi(q)$ and $\pi(r q)=\pi(y)$, since $r q=y$. It follows from the definition of $\bar{\eta}$ that $x \bar{\eta} y=r q$, and the lemma is proved.

Lemma 5.13. Given a congruence $\eta$ on $K$ of finite index there exists a congruence $\eta^{\prime}$ of finite index on $S$ that refines $\eta$, that is, if $x \eta^{\prime} y$ and $x, y \in K$, then $x \eta y$.

Proof. By Lemmas 5.10 and 5.12, $\bar{\eta}$ is a right congruence of finite index on $S$. We claim that $\bar{\eta}$ refines $\eta$. Assume that $x, y \in K$ and $x \bar{\eta} y$. Suppose that $u_{i}, v_{i}, q_{i}$ (where $i=1, \ldots, k)$ are the parameters in a sequence (5.1) connecting $x$ to $y$. By Lemma 5.11, we may assume that $q_{i} \in Q$. Since $x \in K$ and $\pi(x)=\pi\left(q_{1}\right)$, it follows that $q_{1} \in K$. Since $v_{1}, q_{1} \in K$, it follows that their product $v_{1} q_{1} \in K$ and, since $\pi\left(q_{2}\right)=\pi\left(v_{1} q_{1}\right)$, we have $q_{2} \in K$. Continuing this way we see that $q_{i} \in K$ for all $i$. Now since $\eta$ is a congruence on $K$, and all of the parameters $u_{i}, v_{i}$ and $q_{i}$ belong to $K$,

$$
\begin{aligned}
x & =u_{1} q_{1} \eta v_{1} q_{1}=u_{2} q_{2} \eta v_{2} q_{2}=u_{3} q_{3} \eta v_{3} q_{3} \\
& =u_{4} q_{4} \eta \cdots \eta v_{k-1} q_{k-1}=u_{k} q_{k} \eta v_{k} q_{k}=y
\end{aligned}
$$

and so $x \eta y$. Therefore $\bar{\eta}$ is a right congruence on $S$ of finite index refining $\eta$. Now the result follows by setting $\eta^{\prime}=\Sigma(\bar{\eta})$, which by Proposition 5.9 is a finite index congruence on $S$ refining $\eta$. 
THEOREM 5.14. Let $S$ be an inverse semigroup and suppose that $(S, \pi)$ is an extension of $K$ by $T$. If $T$ is finite and $\pi$ is finitely covered, then $S$ is residually finite if and only if $K$ is residually finite.

Proof. The property of being residually finite is inherited by all subsemigroups so, in particular, if $S$ is residually finite, then $K$ is residually finite.

Conversely, assume that $K$ is residually finite and let $x$ and $y$ be arbitrary distinct elements of $S$. If $\pi(x) \neq \pi(y)$, then these two elements are separated by the congruence of finite index associated with $\pi$. Hence assume that $\pi(x)=\pi(y)$. We claim that at least one of the inequalities $x x^{-1} \neq x y^{-1}$ or $y y^{-1} \neq x y^{-1}$ holds. Indeed, if $x x^{-1}=x y^{-1}$, then $x^{-1}=x^{-1} x y^{-1}$, which implies that $x^{-1} \leq y^{-1}$ and consequently $x \leq y$. Similarly, $y y^{-1}=x y^{-1}$ implies $y \leq x$, and the claim follows. Assume that $x x^{-1} \neq x y^{-1}$ (the other case being treated similarly). Now $\pi(x)=\pi(y)$, so $x y^{-1} \in K$. Since $x x^{-1}$ also belongs to $K$, there exists a finite index congruence $\eta$ on $K$ that separates $x x^{-1}$ and $x y^{-1}$. By Lemma 5.13, there exists a congruence $\eta^{\prime}$ of finite index on $S$ that refines $\eta$; in particular $\left(x x^{-1}, x y^{-1}\right) \notin \eta^{\prime}$. Note that $(x, y) \notin \eta^{\prime}$, for otherwise we would have $\left(x^{-1}, y^{-1}\right) \in \eta^{\prime}$, and hence $\left(x x^{-1}, x y^{-1}\right) \in \eta^{\prime}$. Thus $\eta^{\prime}$ has finite index and separates $x$ and $y$, completing the proof.

REMARK 5.15. It is natural to ask whether or not the assumption in the theorem above that $\pi$ is finitely covered is really necessary. We leave this as an open problem.

\section{Homomorphisms with finite kernel}

Let $S$ be an inverse semigroup and suppose that $(S, \pi)$ is an extension of $K$ by $T$. In the previous section, we looked at the relationship between properties of $S$ and those of $K$ under the assumption that $T$ is finite. In this section, we make a few initial observations regarding the study of the relationship between properties of $S$ and those of $T$, under the assumption that $K$ is finite. Recall from the introduction the motivation for doing this: in the case of finitely generated groups these assumptions force $S$ and $T$ to be quasiisometric and hence to share many interesting properties.

The approach here is quite different to that used in previous sections to relate $S$ and $K$. The point here is that when $K$ is finite, it follows that $E(S)$ is finite, and this often allows one to reduce things to the study of maximal subgroups.

We begin with a positive result.

THEOREM 6.1. Let $S$ be an inverse semigroup and suppose that $(S, \pi)$ is an extension of $K$ by $T$. If $S$ is finitely presented and $K$ is finite, then $T$ is finitely presented.

Proof. Since $K$ is finite, it follows that each of $E(S)$ and $E(T)$ is finite. It is well known (see [38, Corollary 4.8]) that an inverse semigroup with finitely many idempotents is finitely presented if and only if all of its maximal subgroups are finitely presented. Let $H$ be an arbitrary group $\mathcal{H}$-class of $T$. Clearly the preimage $\pi^{-1}(H)$ is an inverse subsemigroup of $S$. Let $e$ denote the unique minimal idempotent in the inverse semigroup $\pi^{-1}(H)$, and let $G_{e}$ be the maximal subgroup of $S$ with idempotent $e$. Then it 
is easy to see that the restriction $\psi$ of $\pi$ to $G_{e}$ is a surjective homomorphism from $G_{e}$ onto $H$. However, since $K$ is finite, it follows that the kernel of $\psi$, in the usual group-theoretic sense, is finite. Since $S$ is finitely presented, and $E(S)$ is finite, it follows that the group $G_{e}$ is finitely presented, which, since $\psi: G_{e} \rightarrow H$ is a surjective group homomorphism with finite kernel, implies that $H$ is finitely presented. Since $H$ was arbitrary we conclude that all the maximal subgroups of $T$ are finitely presented, which, since $E(T)$ is finite, implies that $T$ itself is finitely presented.

However, the converse of Theorem 6.1 does not hold, as the following example demonstrates.

Example 6.2. Consider a triple $(G, H, \phi)$ where $G$ is a finitely presented group, $H$ is a group that is not finitely presented, assumed to be disjoint from $G$, and $\phi: H \rightarrow G$ is an embedding. Let $S$ denote the monoid with elements $G \cup H$ and multiplication given by the following rule. Given $x, y \in S$, if $x, y \in H$, then we multiply as in $H$; if $x, y \in G$, then we multiply as in $G$; if $x \in H$ and $y \in G$, then take the product of $\phi(x)$ and $y$ in $G$; if $x \in G$ and $y \in H$, then take the product of $x$ and $\phi(y)$ in $G$. (This is a special case of the classical Clifford semilattice of groups construction; see [26, Section 4.2].)

Define $\pi: S \rightarrow G$ by $\pi(g)=g$ for all $g \in G$, and $\pi(h)=\phi(h)$ for all $h \in H$. Then $(S, \pi)$ is an extension of $K=E(S)$ by $G, K$ is finite and $G$ is finitely presented, but $S$ is not finitely presented since $H$ is not finitely presented, and $H$ is a subsemigroup of $S$ with ideal complement.

On the other hand, in the special case of idempotent separating homomorphisms, the converse does hold.

Theorem 6.3. Let $S$ be an inverse semigroup. Suppose that $(S, \pi)$ is an extension of $K$ by $T$ where $K$ is finite and $\pi$ is idempotent separating. Then $S$ is finitely presented if and only if $T$ is finitely presented.

PRoof. The direct implication was proved in Theorem 6.1.

For the converse, suppose that $T$ is finitely presented. Let $H$ be an arbitrary group $\mathcal{H}$-class in $S$. Since the homomorphism $\pi$ is idempotent separating, it follows that for all $x, y \in S$, we have $x \mathcal{H} y$ in $S$ if and only if $\pi(x) \mathcal{H} \pi(y)$ in $T$. It follows that $H$ is the preimage under $\pi$ of a group $\mathcal{H}$-class $L$ of $T$. Therefore, the restriction $\psi$ of $\pi$ to $H$ defines a surjective group homomorphism $\psi: H \rightarrow L$ with finite kernel. Since $T$ is finitely presented, $L$ is finitely presented, and hence $H$ is finitely presented. Since $H$ was arbitrary, it follows that all maximal subgroups of $S$ are finitely presented, and we conclude that $S$ itself is finitely presented.

6.1. The homological finiteness property $\mathbf{F P}_{\boldsymbol{n}}$. Let $S$ be a monoid and $\mathbb{Z} S$ be the monoid ring over the integers $\mathbb{Z}$. The monoid $S$ is of type left-FP ${ }_{n}$, where $n \geq 0$, if there is a resolution

$$
F_{n} \rightarrow F_{n-1} \rightarrow \cdots \rightarrow F_{1} \rightarrow F_{0} \rightarrow \mathbb{Z} \rightarrow 0
$$


of the trivial left $\mathbb{Z} S$-module $\mathbb{Z}$ in which $F_{0}, F_{1}, \ldots, F_{n}$ are finitely generated free left $\mathbb{Z} S$-modules. For inverse monoids, the properties left-FP ${ }_{n}$ and right-FP ${ }_{n}$ are equivalent, so we simply speak of an inverse monoid of type $\mathrm{FP}_{n}$.

The property $\mathrm{FP}_{n}$ was introduced for groups by Bieri in [6] and since then has received a great deal of attention in the literature; see, for instance, [5, 7, 15, 31]. In monoid and semigroup theory, the property $\mathrm{FP}_{n}$ arises naturally in the study of string rewriting systems (that is, semigroup presentations). A finite complete rewriting system is a finite presentation for a monoid of a particular form (both confluent and Noetherian), which in particular gives a solution of the word problem for the monoid; see [11] for more details. Therefore it is of considerable interest to develop an understanding of which monoids are presentable by such rewriting systems. The connection between complete rewriting systems and homological finiteness properties is given by a result of Anick [3] (see also [12]), which shows that a monoid that admits such a presentation must be of type $\mathrm{FP}_{\infty}$ (meaning type $\mathrm{FP}_{n}$ for all $n$ ). More background on the importance of the property $\mathrm{FP}_{n}$ and other related finiteness conditions in semigroup theory, and the connections with the theory of string rewriting systems, may be found in the survey articles [18, 36].

THEOREM 6.4. Let $S$ be an inverse monoid. If $(S, \pi)$ is an extension of $K$ by $T$ and $K$ is finite, then $S$ is of type $\mathrm{FP}_{n}$ if and only if $T$ is of type $\mathrm{FP}_{n}$.

Proof. It follows from [24, Theorem 3] that an inverse monoid $V$ with a minimal idempotent $e$ has type $\mathrm{FP}_{n}$ if and only if the maximal subgroup $H_{e}$ has type $\mathrm{FP}_{n}$. Let $G_{S}$ and $G_{T}$ be the minimal groups of the monoids $S$ and $T$ respectively. The restriction $\psi$ of $\pi$ to $G_{S}$ is a group homomorphism with finite kernel that maps $G_{S}$ surjectively onto $G_{T}$. It follows that all the following are equivalent: $S$ is of type $\mathrm{FP}_{n} ; G_{S}$ is of type $\mathrm{FP}_{n} ; G_{T}$ is of type $\mathrm{FP}_{n}$ (by the main result of [2]); $T$ is of type $\mathrm{FP}_{n}$.

\section{References}

[1] J. M. Alonso, 'Inégalités isopérimétriques et quasi-isométries', C. R. Acad. Sci. Paris Sér. I Math. 311 (1990), 761-764.

[2] J. M. Alonso, 'Finiteness conditions on groups and quasi-isometries', J. Pure Appl. Algebra 95 (1994), 121-129.

[3] D. J. Anick, 'On the homology of associative algebras', Trans. Amer. Math. Soc. 296 (1986), 641-659.

[4] K. Auinger, 'Residual finiteness of free products of combinatorial strict inverse semigroups', Proc. Roy. Soc. Edinburgh Sect. A 124 (1994), 137-147.

[5] M. Bestvina and N. Brady, 'Morse theory and finiteness properties of groups', Invent. Math. 129 (1997), 445-470.

[6] R. Bieri, Homological Dimension of Discrete Groups, Queen Mary College Mathematics Notes (Mathematics Department, Queen Mary College, London, 1976).

[7] R. Bieri and J. Harlander, 'On the $\mathrm{FP}_{3}$-conjecture for metabelian groups', J. Lond. Math. Soc. (2) 64 (2001), 595-610.

[8] B. Billhardt, 'On a wreath product embedding for regular semigroups', Semigroup Forum 46 (1993), 62-72.

[9] B. Billhardt and I. Szittyai, 'On embeddability of idempotent separating extensions of inverse semigroups’, Semigroup Forum 61 (2000), 26-31. 
[10] J.-C. Birget, S. W. Margolis and J. C. Meakin, 'The word problem for inverse monoids presented by one idempotent relator', Theoret. Comput. Sci. 123 (1994), 273-289.

[11] R. V. Book and F. Otto, String-rewriting Systems, Texts and Monographs in Computer Science (Springer, New York, 1993).

[12] K. S. Brown, 'The geometry of rewriting systems: a proof of the Anick-Groves-Squier theorem', in: Algorithms and Classification in Combinatorial Group Theory (Berkeley, CA, 1989), Mathematical Sciences Research Institute Publications, 23 (Springer, New York, 1992), pp. 137-163.

[13] T. C. Brown, 'Locally finite semigroups', Ukraïn. Mat. Zh. 20 (1968), 732-738.

[14] T. C. Brown, 'An interesting combinatorial method in the theory of locally finite semigroups', Pacific J. Math. 36 (1971), 285-289.

[15] K.-U. Bux and K. Wortman, 'Finiteness properties of arithmetic groups over function fields', Invent. Math. 167 (2007), 355-378.

[16] C. M. Campbell, E. F. Robertson, N. Ruskuc and R. M. Thomas, 'Reidemeister-Schreier type rewriting for semigroups', Semigroup Forum 51 (1995), 47-62.

[17] C. A. Carvalho, 'Presentations of semigroups and inverse semigroups', MSc Thesis, University of St Andrews, 2002.

[18] D. E. Cohen, 'String rewriting and homology of monoids', Math. Structures Comput. Sci. 7 (1997), 207-240.

[19] H. D'Alarcao, 'Idempotent-separating extensions of inverse semigroups', J. Aust. Math. Soc. 9 (1969), 211-217.

[20] P. de la Harpe, Topics in Geometric Group Theory, Chicago Lectures in Mathematics (University of Chicago Press, Chicago, IL, 2000).

[21] T. I. Eršova, 'Inverse semigroups with some finiteness conditions', Izv. Vyssh. Uchebn. Zaved. Mat. 11(186) (1977), 7-14.

[22] È. A. Golubov, 'Finitely approximable regular semigroups', Mat. Zametki 17 (1975), 423-432.

[23] G. M. S. Gomes and M. B. Szendrei, 'Idempotent pure extensions by inverse semigroups via quivers', J. Pure Appl. Algebra 127 (1998), 15-38.

[24] R. Gray and S. J. Pride, 'Homological finiteness properties of monoids, their ideals and maximal subgroups', J. Pure Appl. Algebra, in press.

[25] P.-A. Grillet, Semigroups. An Introduction to the Structure Theory, Monographs and Textbooks in Pure and Applied Mathematics, 193 (Marcel Dekker Inc., New York, 1995).

[26] J. M. Howie, Fundamentals of Semigroup Theory, London Mathematical Society Monographs. New Series, 12 (Oxford University Press, New York, 1995).

[27] S. V. Ivanov, S. W. Margolis and J. C. Meakin, 'On one-relator inverse monoids and one-relator groups', J. Pure Appl. Algebra 159 (2001), 83-111.

[28] M. V. Lawson, Inverse Semigroups. The Theory of Partial Symmetries (World Scientific, River Edge, NJ, 1998).

[29] M. V. Lawson, S. W. Margolis and B. Steinberg, 'Expansions of inverse semigroups', J. Aust. Math. Soc. 80 (2006), 205-228.

[30] B. Le Saëc, J.-E. Pin and P. Weil, 'Semigroups with idempotent stabilizers and applications to automata theory', Internat. J. Algebra Comput. 1 (1991), 291-314.

[31] I. J. Leary and M. Saadetoğlu, 'Some groups of finite homological type', Geom. Dedicata 119 (2006), 113-120.

[32] R. C. Lyndon and P. E. Schupp, Combinatorial Group Theory, reprint of the 1977 edition Classics in Mathematics (Springer, Berlin, 2001).

[33] W. Magnus, A. Karrass and D. Solitar, Combinatorial Group Theory, Presentations of Groups in Terms of Generators and Relations, revised edn (Dover Publications, New York, 1976).

[34] J. C. Meakin and A. Yamamura, 'Bass-Serre theory and inverse monoids', in: Semigroups and Applications (St. Andrews, 1997) (World Scientific, River Edge, NJ, 1998).

[35] W. D. Munn, 'Free inverse semigroups', Proc. Lond. Math. Soc. 329 (1974), 385-404. 
[36] F. Otto and Y. Kobayashi, 'Properties of monoids that are presented by finite convergent stringrewriting systems-a survey', in: Advances in Algorithms, Languages, and Complexity (Kluwer, Dordrecht, 1997), pp. 225-266.

[37] A. Ya. Ovsyannikov, 'A general theorem on inverse semigroups with finiteness conditions', Mat. Zametki 41 (1987), 138-147, 285.

[38] N. Ruškuc, 'Presentations for subgroups of monoids', J. Algebra 220 (1999), 365-380.

[39] N. Ruškuc and R. M. Thomas, 'Syntactic and Rees indices of subsemigroups', J. Algebra 205 (1998), 435-450.

[40] B. M. Schein, 'Free inverse semigroups are not finitely presentable', Acta Math. Acad. Sci. Hung. 26 (1975), 41-52.

[41] B. Steinberg, 'A topological approach to inverse and regular semigroups', Pacific J. Math. 208 (2003), 367-396.

[42] B. Steinberg, 'The uniform word problem for groups and finite Rees quotients of $E$-unitary inverse semigroups', J. Algebra 266 (2003), 1-13.

[43] B. Steinberg, 'A groupoid approach to discrete inverse semigroup algebras', Adv. Math. 223 (2010), 689-727.

[44] J. B. Stephen, 'Presentations of inverse monoids', J. Pure Appl. Algebra 63 (1990), 81-112.

CATARINA CARVALHO, Centro de algebra da Universidade de Lisboa, Av. Prof. Gama Pinto 2, 1649-003 Lisboa, Portugal

e-mail: ccarvalho@cii.fc.ul.pt

ROBERT D. GRAY, Centro de algebra da Universidade de Lisboa, Av. Prof. Gama Pinto 2, 1649-003 Lisboa, Portugal

e-mail: rdgray@fc.ul.pt

NIK RUSKUC, School of Mathematics and Statistics, University of St Andrews, St Andrews KY16 9SS, UK

e-mail: nik@mcs.st-andrews.ac.uk 